THE ASTROPHYSICAL JOURNAL, 470:301-321, 1996 October 10

1996. The American Astronomical Society. All rights reserved. Printed in U.S.A.

\title{
032462 RESULTS OF DETAILED MODELING OF THE NARROW-LINE REGION OF SEYFERT GALAXIES
}

\author{
David MOORE AND RosS D. COHEN \\ Center of Astrophysics and Space Sciences 0111, 9500 Gilman Drive, University of California, San Diego, La Jolla, CA 92093-0111 \\ Received 1995 February 22; accepted 1996 April 23
}

\begin{abstract}
We present model line profiles of [O II] $\lambda 3727,[\mathrm{Ne}$ III] $\lambda 3869,[\mathrm{O}$ III $] \lambda 5007,[\mathrm{Fe} \mathrm{VII}] \lambda 6087,[\mathrm{Fe} \mathrm{x}]$ $\lambda 6374,[\mathrm{O} \mathrm{I}] \lambda 6300, \mathrm{H} \alpha \lambda 6563$, and $[\mathrm{S} \mathrm{II}] \lambda 6731$. The profiles presented here illustrate explicitly the pronounced effects that collisional de-excitation, and that spatial variations in both the ionization parameter and cloud column density, have on narrow-line region (NLR) model profiles. The above effects were included only qualitatively in a previous analytical treatment by Moore \& Cohen.

By making a direct correspondence between these model profiles and the analytical model profiles of Moore \& Cohen, and by comparing with the observed profiles presented in a companion paper and also with those presented elsewhere in the literature, we strengthen some of the conclusions of Moore \& Cohen. Most notably, we argue for constant ionization parameter, uniformly accelerated outflow of clouds that are individually stratified in ionization, and the interpretation of emission-line width correlations with ionization potential as a column density effect. For comparison with previous observational studies, such as our own in a companion paper, we also calculate profile parameters for some of the models, and we present and discuss the resulting line width correlations with critical density $\left(n_{\mathrm{cr}}\right)$ and ionization potential (IP). Because the models we favor are those that produce extended profile wings as observed in high spectral resolution studies, the line width correlations of our favoured models are of particular interest. Line width correlations with $n_{\mathrm{cr}}$ and/or IP result only if the width parameter is more sensitive to extended profile wings than is the full width at half-maximum (FWHM). Correlations between FWHM and $n_{\mathrm{cr}}$ and/or IP result only after convolving the model profiles with a broad instrumental profile that simulates the lower spectral resolution used in early observational studies.

The model in agreement with the greatest number of observational considerations has electron density decreasing outward from $n_{e} \approx 10^{6} \mathrm{~cm}^{-3}$ to $n_{e} \approx 10^{2} \mathrm{~cm}^{-3}$ and, due to collisional de-excitation effects in the lowest velocity clouds, it generates broad flat-topped profile peaks in the lines of lowest critical density (e.g., [O II] $\lambda 3727$ and [S II] $\lambda \lambda 6716,6731$ ). Because the observed profile peaks of both low and high critical density lines are often very similar, our favored model requires a contribution to NLR emission-line spectra from low-velocity, low-density, and low-ionization gas not included in the model NLR. Subject headings: galaxies: nuclei — galaxies: Seyfert — line: profiles
\end{abstract}

\section{INTRODUCTION}

The models presented in this paper are intended primarily to complement observations presented in a companion paper (Moore, Cohen, \& Marcy 1996, hereafter Paper I). The models are also suitable for comparison with other observational studies, such as those of De Robertis \& Osterbrock $(1984,1986)$ and Veilleux (1991a). In this paper, we illustrate the pronounced effects that collisional deexcitation and spatial variations in both the ionization parameter $(\Gamma)$ and cloud column density $\left(N_{\mathrm{col}}\right)$ throughout the narrow-line region (NLR) have on NLR model profiles. By comparing the model profiles with those observed, we infer the extent to which these effects are likely important in the observed profiles presented in Paper I and elsewhere. To strengthen further the connection with previous observational studies, we also calculate profile parameters for some of the models, and we present and discuss the resulting line width correlations with critical density $\left(n_{\mathrm{cr}}\right)$ and ionization potential (IP) in $\S 5$.

The first treatment of NLR kinematics that allowed for gradients in both the electron density and the cloud velocity across the NLR, and which included photoionization calculations rather than making very simple assumptions regarding emission-line luminosity across the NLR, was that of Whittle (1982). Since Whittle's work, there have been two important observational results adding new constraints on
NLR models, suggesting that a new treatment of NLR kinematics is appropriate.

First, the very high dispersion $\left(10 \mathrm{~km} \mathrm{~s}^{-1}\right)$ and high signal-to-noise ratio (S/N) profiles of Veilleux (1991a) show well-resolved extended wings. This requires not only line emission over a wide range of cloud velocity, but also a shallow radial dependence for the line emission so that neither the lowest nor the highest radial velocity clouds dominate the contribution to the line profile (see Moore \& Cohen 1994, hereafter MC). Second, we showed in Paper I that a wide range in velocity can be inferred from both low and high $n_{\mathrm{cr}}$ lines and from lines with very different sensitivity to ionization parameter. Together, these two observational results greatly constrain NLR models. In fact, the models discussed here are somewhat overconstrained in that no single model can satisfy all the observationally imposed constraints. Specifically, the model that is consistent with the greatest number of observational considerations cannot account for the fact that, in some objects, the similarity in profiles of both low and high $n_{\mathrm{cr}}$ lines applies to the profile cores as well as the wings. This is discussed further in $\$ 3$ and 6 .

\section{CAlCulating THE LINE PROFILES}

In this section, we describe our procedure for calculating line profiles. In Appendix A of MC, we described this pro- 
cedure for the purpose of comparison with the analytical model profiles presented there. An appropriate portion of the description is summarized here for convenient reference.

A spherically symmetric NLR is assumed in all the following models, even though biconical emission is clearly resolved in some nearby narrow-line regions (NLRs). In $\mathrm{MC}$, we showed that the profile wings are not very sensitive to either the opening angle or to the orientation of a biconical structure containing all the observed line emission, provided we rescale the velocity field so that the maximum cloud velocity that contributes to the line profile is fixed. Thus, as long as we are cautious regarding inferences based on the observed profile cores, the results of our spherically symmetric models are applicable to a biconical NLR. Little information is lost by considering only spherically symmetric models and the corresponding profile wings because it is unrealistic to assume purely radial cloud flow for the lowest as well as the highest velocity clouds. In this paper, we will also demonstrate that the predicted effects of spatial variations in the electron density, ionization parameter, and column density are sufficiently pronounced that our most important conclusions will not likely be invalidated by including secondary, nonradial components of the velocity field.

In these models, we incorporate explicit photoionization calculations to derive line profiles of $[\mathrm{O}$ II] $\lambda 3727,[\mathrm{Ne}$ III] $\lambda 3869,[\mathrm{O} \mathrm{mI}] \lambda 5007,[\mathrm{Fe} \mathrm{VII}] \lambda 6087,[\mathrm{Fe} \mathrm{x}] \lambda 6374,[\mathrm{O} \mathrm{I}]$ $\lambda 6300, \mathrm{H} \alpha \lambda 6563$, and [S II] $\lambda 6731$. For each line, the following integral is evaluated numerically:

$$
\begin{aligned}
I\left(v_{z}\right)= & \int_{r_{\min } / r_{\max }}^{1} \sigma[n(x), \Gamma(x)] \\
& \times \frac{s^{2}(x) n_{\mathrm{cl}}(x)}{v(x)} \Theta\left[\frac{v_{z}}{v(x)}\right] \text { obsc }\left[\mu\left(v_{z}, x\right), x\right] x^{2} d x .
\end{aligned}
$$

In equation (1), $s$ is the cloud size, $n$ is the proton number density at the front of the cloud, $n_{\mathrm{cl}}$ is the number of clouds per unit volume throughout the NLR, $v_{z}$ is the measured projected velocity of interest, and $v(x)$ is the radial velocity. The cosine of the polar angle is $\mu\left(v_{z}, x\right)$, which equals $v_{z} / v(x)$, as follows from the delta function that selects the projected velocity of interest (MC), and $\Theta=1$ if its argument is less than 1 in absolute value and 0 otherwise. Here $\Gamma$ is the ionization parameter. As in MC, we adopt Ferland's (1989) definition of the dimensionless ionization parameter, $\Gamma$, as essentially the ratio of incident ionizing photons to protons. Formally,

$$
\Gamma=\frac{\int_{v_{0}}^{\infty}\left(L_{v} / h v\right) d v}{4 \pi n_{\mathrm{H}} r^{2} c},
$$

where $v_{0}$ is the threshold for ionizing hydrogen, $n_{\mathrm{H}}$ is the proton number density (the definition includes both ionized and neutral hydrogen), and $r$ is the distance from the photoionizing source.

The flux, $\sigma$, into each emission line is determined from a two-dimensional quadratic spline fit to line strengths computed using the photoionization code CLOUDY, kindly provided by G. Ferland. We assumed stratified clouds for all models in the two-dimensional grid (see MC for more details). As in Paper I, we use the term "stratified cloud" to denote a cloud that is individually stratified in ionization. We assume $s^{2}(x) \propto n^{-2 / 3}(x)$ (constant mass spheres), $n_{\mathrm{cl}} \propto$ $1 /\left[x^{2} v(x)\right]$ (continuity), and that the cloud velocity is $v(x) \propto x^{\alpha}$.

The quantity obsc $\left[\mu\left(v_{z}, x\right), x\right]$, is a multiplicative factor, determined from expressions derived elsewhere (Moore 1994, 1995), that allows for the effects of both internal and external obscuration by dust. To treat internal obscuration, we assumed that dust is distributed uniformly throughout a cloud and that the line emission is uniform throughout a crescent in the interior of the cloud. The size of the crescent was calculated using CLOUDY. To treat external obscuration, we assumed that dust was distributed throughout the intercloud medium with a power-law radial dependence.

In no case were the blue wings of the profiles very sensitive to details of the obscuration. Therefore, to reduce the number of profiles, we restrict the model profiles presented here to those in which obscuration is not included. (We do, however, include one model in which the treatment of obscuration is identical to that of Vrtilek [1985].) The conclusions of this paper are based largely on comparing the blue wings of the model profiles to those observed. Because obscuration is not included in the models presented here, they cannot produce correlations of line shift with IP and/or $n_{\text {cr }}$ and cannot account for the observed preponderance of blueward asymmetries. Nevertheless, we have calculated profiles for models that include realistic treatments of obscuration, and these models do produce blueward asymmetric profiles and line shift correlations with IP and $n_{\mathrm{cr}}$. The obscuration is mostly internal or external to the emitting clouds according to whether the direction of cloud flow is inward or outward, respectively. The models we calculated in which obscuration was included have some interesting implications for the nature of the dust distribution, but they are not relevant to the conclusions of this paper (with the very important exception, of course, that the blue wings of the model profiles are similar to those in which obscuration is neglected). In the case of inflow, the blueward asymmetry requires stratified clouds. For outflow, the clouds may be stratified or or unstratified, even though the obscuration is mostly external in either case. (Stratified clouds may be optically thick to the incident photoionizing continuum, but optically thin to the line emission.)

We define $N_{\text {col }} / N_{\min }$ as the column density in units of the minimum column density needed for an [ $\left[\begin{array}{ll}\mathrm{O} & 1\end{array}\right] \lambda 6300$ emitting zone to exist in the back of the clouds. In some models, $N_{\text {col }} / N_{\min }$ changes in a manner that is inconsistent with the assumption, under which $\sigma$ was calculated, of stratified clouds throughout the NLR. To make the appropriate correction when $N_{\text {col }}<N_{\text {min }}$ (not completely stratified clouds), we calculate the factor by which $\sigma s^{2}$ (see eq. [1]) overestimates a cloud's contribution to the line profile. We formally define $r_{s}$ as the depth at which hydrogen is $50 \%$ ionized and $r_{\mathrm{fe}}$ as the depth at which the [Fe vII] $\lambda 6087$ and [Fe x] 26374 lines reach $99 \%$ of their final strength. If $s<r_{\mathrm{fe}}$, we rescale the Fe line strengths by the factor, $s / r_{\mathrm{fe}}$ and set all other line strengths to zero. For $r_{\mathrm{fe}}<s<r_{s}$, the $\mathrm{Fe}$ line strengths are unchanged, [O $\mathrm{OII}] \lambda 5007$ and [Ne III] $\lambda 3869$ are rescaled by the factor, $\left.\left(s-r_{\mathrm{fe}}\right) / r_{s}-r_{\mathrm{fe}}\right)$, and the [O II] $\lambda 3727,\left[\mathrm{O}_{\mathrm{I}}\right] \lambda 6300$, and $[\mathrm{S} \mathrm{II}] \lambda 6731$ line strengths are set to zero. Assuming uniform emissivity in the relevant zone of the cloud, the emission-line flux from a spherical cloud is of the form

$$
I=\pi\left(\delta-\frac{1}{12} \delta^{3}\right),
$$

where $\delta$ is the depth of the emitting crescent relative to the cloud radius (see $\mathrm{MC}$ ). The preceding scale factors are valid to the extent that the second term in the right-hand side of equation (2) is negligible.

Now we present and discuss the grid of detailed kine- 
matical models. The profile we denote as [O II $] \lambda 3727$ is actually that of $[\mathrm{O}$ II] $\lambda 3726$; it is not the blend, $\lambda 3726+\lambda 3729$. The $\left[\mathrm{O}_{1}\right] \lambda 6300$ and $\left[\mathrm{S}_{\mathrm{II}}\right] \lambda 6731$ profiles are to be interpreted with caution because the flux in these lines depends on the cloud column density, which was not corrected as above for violating the assumption of constant mass in the grid of static models. As discussed in MC, the fact that emission into the ionized lines changes little as $N_{\text {col }}$ is increased above $N_{\min }$ was exploited by fixing $N_{\text {col }}=N_{\text {min }}$ $+\epsilon$, where $\epsilon<0.1$ dex but is otherwise arbitrary, to reduce the dimensionality of the grid of static models from three to two $(\Gamma$ and $n)$. Although the variation of profile flux with decreasing projected velocity in the $\left[\mathrm{O}_{\mathrm{I}}\right] \lambda 6300$ and $[\mathrm{S} \mathrm{II}]$ $\lambda 6731$ model profiles is not accurate in detail, comparison of the two profiles illustrates the effects of collisional deexcitation because these two lines have very different $n_{\mathrm{cr}}$. [O II] $\lambda 3727$ can illustrate also the effects of collisional deexcitation, with the added advantage that the emission from a single cloud into this line is not sensitive to the amount of gas with $N_{\text {col }}>N_{\min }$.

Results of models with cloud velocity increasing outward are presented in $\S 3$, and results of models with velocity increasing inward are presented in $\S 4$. In all the following models, we normalize the density profile throughout the NLR such that $n_{\min } \equiv n\left(r=r_{\max }\right)=10^{2} \mathrm{~cm}^{-3}$. This ensures that the constraints of low density, implied by [S II] $\lambda \lambda 6716$, 6731 and [O II] $\lambda 3727$, can be satisfied. In some of the following models, we violate the constraint of high density, inferred from the [O III] lines, $\lambda 4959, \lambda 5007$, and $\lambda 4363$ (see $\S 5$ ), by setting $n_{\max }=10^{4} \mathrm{~cm}^{-3}$ rather than $n_{\max } \gtrsim 10^{6}$ $\mathrm{cm}^{-3}$. The velocity field is normalized to a maximum value of $500 \mathrm{~km} \mathrm{~s}^{-1}$. Neglecting the effects of obscuration, we can then parameterize a model NLR by $\alpha, \gamma, \Gamma\left(r=r_{\max }\right), r_{\min }$, and $N_{\text {col }}\left(r=r_{\min }\right)$, where $v \propto r^{x}$ and $n \propto r^{y}$. The parameters for each of these models are listed in Table 1 .

Interpreting the profiles is facilitated by comparing with the results of the simple analytical treatment of MC. In MC we derived the following analytical expression for line profiles:

$$
I\left(v_{z}\right)=\left\{\begin{array}{cc}
0 & \text { if }\left|v_{z}\right| \geq v_{\max }, \\
I_{\text {wing }} & \text { if } v_{\min } \leq\left|v_{z}\right| \leq m_{\max }, \\
I_{\text {core }} & \text { if } 0 \leq\left|v_{z}\right| \leq n_{\min },
\end{array}\right.
$$

where (after normalizing the peak to unity)

$$
I_{\text {wing }}=\left[1-\left(\frac{v_{\min }}{v_{\max }}\right)^{\beta}\right]^{-1}\left[1-\left(\frac{\left|v_{z}\right|}{v_{\max }}\right)^{\beta}\right],
$$

$$
\begin{aligned}
& \beta=\left(\alpha^{\prime}+1\right) / \alpha, \text { and } \\
& \qquad I_{\text {core }}=I_{\text {wing }}\left(v_{z}=v_{\text {min }}\right)=1 .
\end{aligned}
$$

The measured projected velocity of interest is $v_{z}$, and $v_{\min }$ and $v_{\max }$ are the minimum and maximum radial velocity in the NLR, respectively. The above assumes a velocity law, $v \propto \boldsymbol{r}^{\alpha}$, and an emissivity law, $E \propto \boldsymbol{r}^{\alpha^{\prime}}$, where $E$ is the line emission in units of ergs $\mathrm{s}^{-1} \mathrm{~cm}^{-3}$. This expression yields profiles with flat-topped profile peaks of half-width $v_{\min }$. For the particular case of constant-mass spherical clouds of constant ionization parameter obeying the equation of continuity,

$$
\beta=\frac{1}{3 \alpha}-2 .
$$

The model profiles are presented in Figures 1-13. The solid line in each figure represents a model profile after convolution with a $\sigma=10 \mathrm{~km} \mathrm{~s}^{-1}$ Gaussian, corresponding to the level of thermal broadening of $10^{4} \mathrm{~K}$ gas, and thus to the highest practical spectral resolution of any observational study. The dashed lone represents a model profile after convolution with a $\sigma=32 \mathrm{~km} \mathrm{~s}^{-1}$ Gaussian, corresponding to the spectral resolution in the red of our own study (Paper I).

\section{RESULTS FOR MODELS WITH $v$ INCREASING OUTWARD}

Model 1.-We begin by presenting a model that replicates the observed extended profile wings. This model has $\alpha=1(v \propto r)$ and $\gamma=-2$ (constant ionization parameter). The ionization parameter is one throughout this NLR. We choose an ionization parameter somewhat higher than the value we argue for in MC so that this model corresponds as closely as possible with models in which $\Gamma$ decreases outward (for later comparison [e.g., models 7 and 8]). We set $r_{\min }=22.5 \mathrm{pc}$ and $N_{\mathrm{col}}\left(r=r_{\min }\right)=10^{24} \mathrm{~cm}^{-2}$. The column density at $r=r_{\min }$ is higher than what is actually required to maintain an $\left[\mathrm{O}_{\mathrm{I}}\right]$ zone throughout this NLR in order to correspond as closely as possible to a larger model NLR in which an outwardly decreasing $N_{\text {col }}$ requires a higher $N_{\text {col }}$ at $r=r_{\min }$ (e.g., model 3). There is no obscuration in this model. Note that $n_{\min }$ and $\Gamma\left(r=r_{\max }\right)$ fix $r_{\max }$ at $225 \mathrm{pc}$, and $n_{\min }, \gamma$, and $r_{\min } / r_{\max }$ fix $n\left(r=r_{\min }\right) \equiv n_{\max }$ at $10^{4}$ $\mathrm{cm}^{-3}$.

The resulting line profiles are presented in Figure 1. This model, which corresponds to an analytical model with $\beta=-1.7$ (see MC) and $v_{\min }=50 \mathrm{~km} \mathrm{~s}^{-1}$, produces profiles

TABLE 1

Detailed Kinematical Models at a Glance

\begin{tabular}{cccccccccc}
\hline \hline Model & $\begin{array}{c}r_{\min } \\
(\mathrm{pc})\end{array}$ & $\begin{array}{c}r_{\max } \\
(\mathrm{pc})\end{array}$ & $\begin{array}{c}n_{\max } \\
\left(\mathrm{cm}^{-3}\right)\end{array}$ & $\alpha$ & $\gamma$ & $\Gamma\left(r_{\text {min }}\right)$ & $\Gamma\left(r_{\text {max }}\right)$ & $\begin{array}{c}N_{\text {col }}\left(r_{\text {min }}\right) \\
\left(\mathrm{cm}^{-2}\right)\end{array}$ & $\begin{array}{c}N_{\text {col }}\left(r_{\text {max }}\right) \\
\left(\mathrm{cm}^{-2}\right)\end{array}$ \\
\hline $1 \ldots \ldots$ & 22.5 & 225 & $10^{4}$ & 1 & -2 & 1 & 1 & $10^{24}$ & $5 \times 10^{22}$ \\
$2 \ldots \ldots$ & 22.5 & 225 & $10^{4}$ & 0.5 & -2 & 1 & 1 & $10^{24}$ & $5 \times 10^{22}$ \\
$3 \ldots \ldots$ & 4.1 & 411 & $10^{6}$ & 0.5 & -2 & 0.3 & 0.3 & $2 \times 10^{24}$ & $4 \times 10^{21}$ \\
$4 \ldots \ldots$ & 4.1 & 411 & $10^{6}$ & 0.5 & -2 & 0.3 & 0.3 & $5 \times 10^{23}$ & $10^{21}$ \\
$5 \ldots \ldots$ & 16.4 & 411 & $6 \times 10^{4}$ & 0.5 & -2 & 0.3 & 0.3 & $10^{23}$ & $10^{21}$ \\
$6 \ldots \ldots$ & 16.4 & 411 & $6 \times 10^{4}$ & 0.5 & -2 & 0.3 & 0.3 & $10^{23}$ & $10^{21}$ \\
$7 \ldots \ldots$ & 71 & 710 & $10^{3}$ & 1 & -1 & 1 & 0.1 & $10^{23}$ & $2 \times 10^{22}$ \\
$8 \ldots \ldots$ & 22.5 & 2245 & $10^{4}$ & 1 & -1 & 1 & 0.01 & $10^{23}$ & $5 \times 10^{21}$ \\
$9 \ldots \ldots$ & 22.5 & 2245 & $10^{4}$ & -0.5 & -1 & 1 & 0.01 & $3 \times 10^{22}$ & $1.5 \times 10^{21}$ \\
$10 \ldots \ldots$ & 2.25 & 22450 & $10^{6}$ & -0.5 & -1 & 1 & $10^{-4}$ & $3 \times 10^{22}$ & $7 \times 10^{19}$ \\
$11 \ldots \ldots$ & 4.1 & 411 & $10^{6}$ & -0.5 & -2 & 0.3 & 0.3 & $3 \times 10^{24}$ & $7 \times 10^{21}$ \\
$12 \ldots \ldots$ & 4.1 & 411 & $10^{6}$ & -0.5 & -2 & 0.3 & 0.3 & $10^{23}$ & $2 \times 10^{20}$ \\
$13 \ldots \ldots$ & 7.1 & 710 & $10^{5}$ & -0.5 & -1.5 & 1 & 0.1 & $10^{24}$ & $10^{22}$ \\
\hline
\end{tabular}



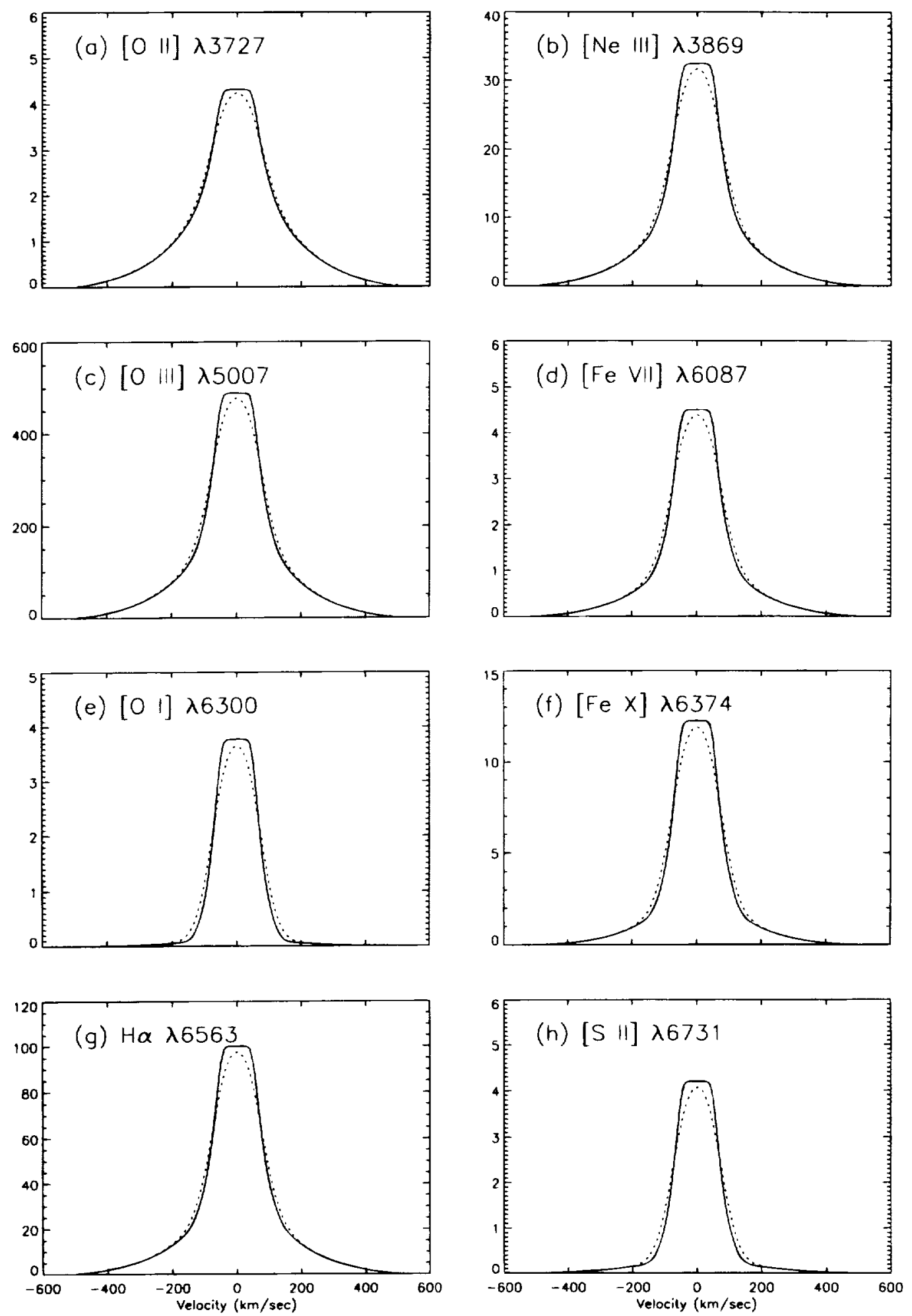

Fig. 1.- The line profiles of model 1, an outflow model with constant ionization parameter. Specific model parameters are provided in Table 1. This model replicates the observed extended profile wings but does not include $n_{e} \geq 10^{6} \mathrm{~cm}^{-3}$ gas, as required by the [O m] lines in many objects. Solid lines represent the model profiles after convolution with a $\sigma=10 \mathrm{~km} \mathrm{~s}^{-1}$ Gaussian, corresponding to thermal broadening of $T=10^{4} \mathrm{~K}$ gas. Dashed lines represent the model profiles after convolution with a $\sigma=32 \mathrm{~km} \mathrm{~s}^{-1}$ gas, corresponding to the spectral resolution in the red of our own study (Paper I).

and relative line strengths (other than the $[\mathrm{O} \mathrm{III}] \lambda 4363$ to [O III] $\lambda 5007$ line strength ratio) that agree with observations about as well as any model discussed in this paper. However, the range in density and radial coordinates encompassed by this model NLR is small. A maximum density of $10^{4} \mathrm{~cm}^{-3}$ would require an alternative to photo- ionization to account for relatively weak $[\mathrm{O} \mathrm{II}] \lambda 5007 \mathrm{emis}-$ sion relative to $\lambda 4363$, seen in many objects. Note that [O II] 23727 is slightly broader than higher $n_{\mathrm{cr}}$ lines because collisional de-excitation effects are becoming important in the lowest velocity clouds even with $n_{\max }$ as low as $10^{4}$ $\mathrm{cm}^{-3}$. 

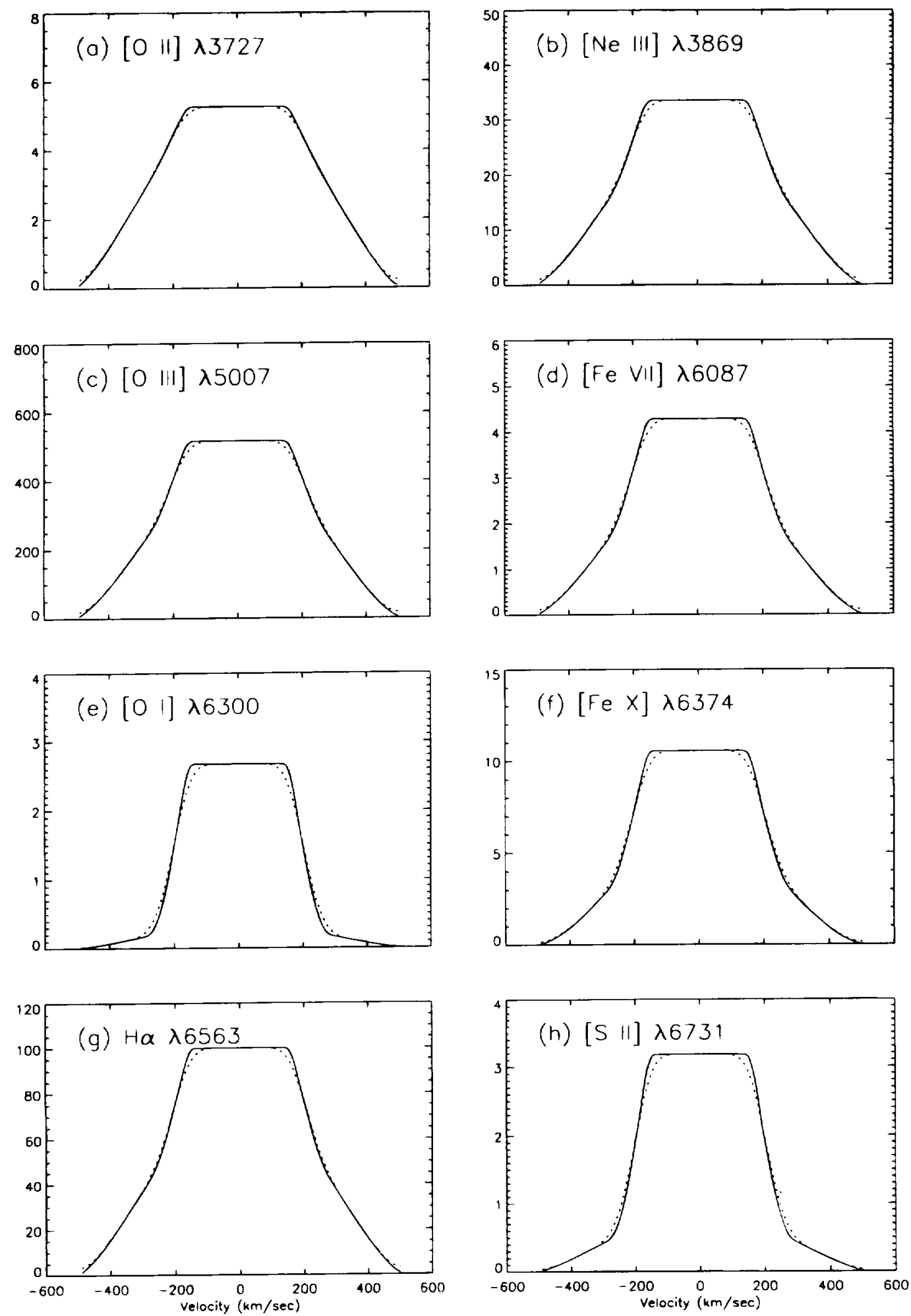

Fig. 2-- The line profiles of model 2, an outflow model. This model is identical to model 1 except that the velocity field is less steep. Specific model parameters are provided in Table 1 . The model includes only a narrow range of radial velocity and profiles not very different from boxcar profiles result.

Model 2.-The second model (Fig. 2) is presented to illustrate how a substantial range of radial velocity can be inferred directly by visual inspection of the profiles. This model is identical to the first except that $\alpha=0.5\left(v \propto r^{1 / 2}\right)$. Model 2 corresponds to an analytical model with $\beta=-1.3$ and $v_{\min }=160 \mathrm{~km} \mathrm{~s}^{-1}$. As was evident in the grid of analytical models (MC), as emission into the line profiles begins to include only a narrow range in cloud velocity, the resulting profiles begin to resemble boxcar profiles.

Model 3.-We consider model 3 as (Fig. 3) as the prototype of a model that shows correlations of line width with neither ionization potential nor critical density (see $\$ 5$ ). The profiles of model 3 are very different from those of model 2 because model 3 encompasses a much wider range of radial 

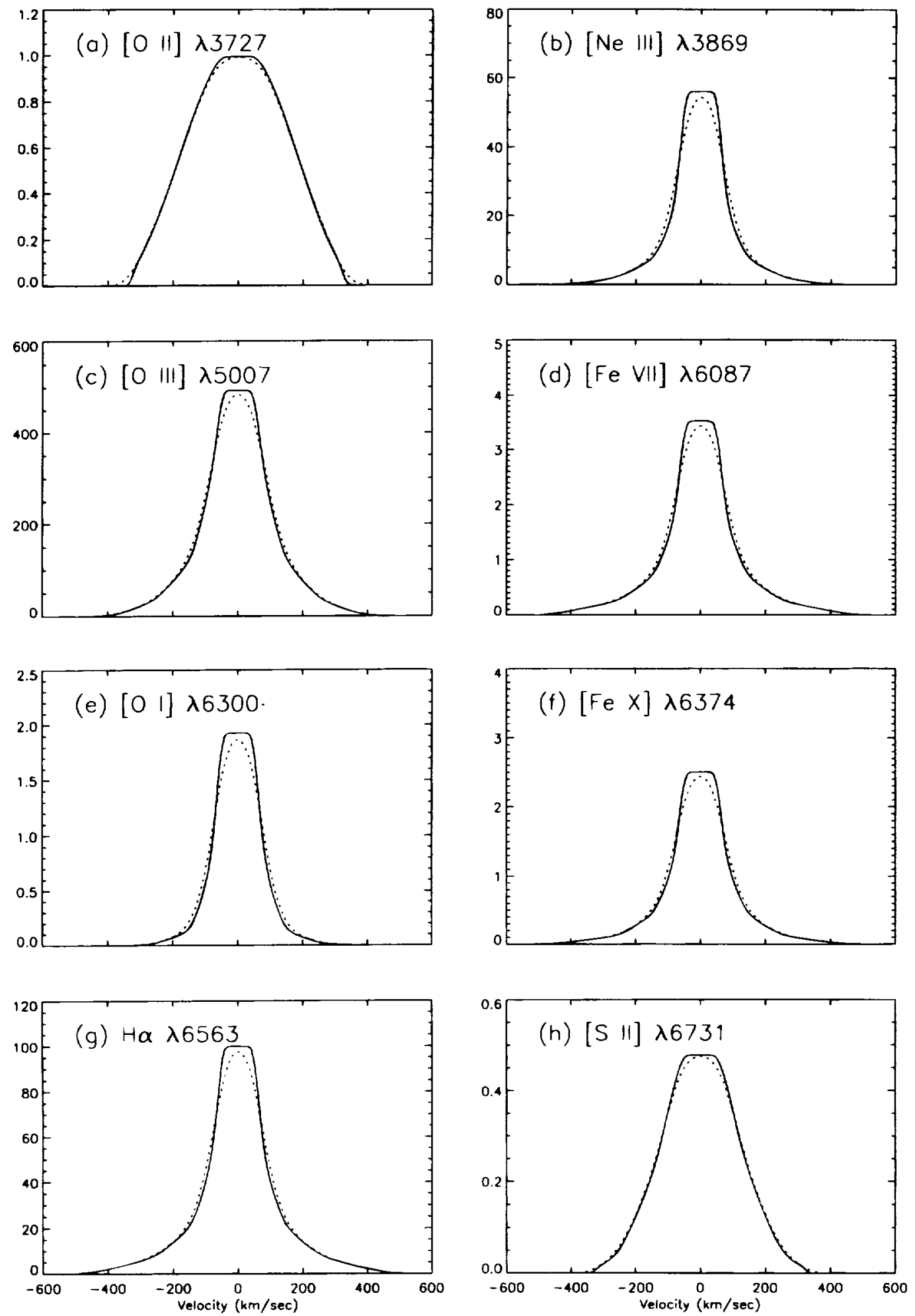

FIG. 3.-The line profiles of model 3, an outflow model. This model includes a wider range of cloud velocities than does model 2 , resulting in more pronounced wings in the former. Model 3 is otherwise identical to model 2 except for a modest increase in the cloud column density and a modest decrease in the ionization parameter, the latter achieved by increasing slightly the distance between the NLR and the central source. Specific model parameters are provided in Table 1. We postulate that model 3 is representative of objects that do not show correlations of line width with ionization potential. Note that this model cannot account for the similarity in profiles of lines with low and high critical density, observed in some objects.

velocity. Here $v \propto r^{1 / 2}$ and $\Gamma=0.3$ throughout the NLR. The profiles of model 3 are similar to those of model 1 in that both models encompass similar ranges of velocity. The difference is that the range of velocity in model 1 is sampled over a narrow range of radial coordinates and the con- straint of high-density gas is violated.

In model 3, the electron density decreases outward from $10^{6}$ to $10^{2} \mathrm{~cm}^{-3}$, while $N_{\text {col }}$ decreases outward from $2 \times 10^{24} \mathrm{~cm}^{-2}$ to $4 \times 10^{21} \mathrm{~cm}^{-2}$. Note, however, the effects of collisional de-excitation in the core of [O II] $\lambda 3727$ (as 
well as of [ $\mathrm{S} \mathrm{II}_{\mathrm{I}}$ 26731). This emission line is inconsistent in strength and in shape with that observed. If the [O III] lines result from photoionization, which requires the presence of $n \gtrsim 10^{6} \mathrm{~cm}^{-3}$ gas in at least some objects, then this difficulty is not easily avoided, however. The constraint of highdensity gas is discussed in more detail in $\S 6$.

Note that the wings of [ $\mathrm{Ne}$ III] $\lambda 3869$ and of [O III] $\lambda 5007$ are slightly less extended than those of $[\mathrm{Fe} \mathrm{VII}] \lambda 6087$ and of [Fe x] $\lambda 6374$. This is because the cloud column density decreases to $N_{\text {col }}<N_{\min }$ slightly before the clouds reach their maximum velocity at the outer NLR radius. The effect can be made either more or less pronounced by varying $N_{\mathrm{col}}$ by factors of order unity.

Model 4.- The fourth model (Fig. 4) is the same as the third, except that now we have decreased the column density of all NLR clouds by a factor of 4 , and correlations of line width with ionization potential result ( $\$ 5$ ). In this model, $N_{\mathrm{col}} / N_{\min }$ decreases to less than 1 well before the clouds reach their maximum velocity, as is clear from the absence of extended profile wings in all but the $\mathrm{Fe}$ lines. Note that a line width correlation with ionization potential would result for a width parameter that is sensitive to the wings, but not necessarily for the FWHM. For example, the FWHMs of [O III] $\lambda 5007$ and of [Fe viI] $\lambda 6087$ are nearly identical to each other in models 3 and 4 , even though the full width at zero intensity (FWZI) of $\lambda 6087$ is significantly larger than that of $\lambda 5007$ for model 4 .

We postulate that model 4 , in which $N_{\text {col }}$ decreases outward to less than $N_{\min }$ well before the clouds reach their maximum velocity at the outermost radius, is representative of objects that show correlations of line width with ionization potential, and model 3, in which $N_{\mathrm{col}}>N_{\text {min }}$ throughout the NLR, is representative of objects that do not. Model 3 can account for the observed profiles of MCG 8-11-11 and NGC 4151 discussed in Paper I (no line width correlations), while model 4 can account for the profiles of Mrk 79, Mrk 704, and Mrk 841 (the extent of the wings increases systematically with increasing ionization potential).

Models 5 and 6.-Models 5 (Fig. 5) and 6 are presented to illustrate how FWHM correlations with $I p / n_{\mathrm{cr}}$ might result from low spectral resolution studies but not from high spectral resolution studies. These models include a somewhat lesser range of radial coordinates than do models 3 and 4 . In addition, we have added a dusty intercloud medium (ICM) with an optical depth of 1 across the entire NLR. The treatment of obscuration here is identical to that of Vrtilek (1985).

Model 6 (Fig. 6) is identical to model 5, but we have convolved the resulting profiles with a Gaussian with FWHM $=150 \mathrm{~km} \mathrm{~s}^{-1}(2.5 \AA$ at $5000 \AA)$ to illustrate the effects of decreased spectral resolution. Note that the difference in FWHM between [O III] $\lambda 5007$ and [Fe VII] $\lambda 6087$ is accentuated by the convolution. The FWHM after convolution is $275 \mathrm{~km} \mathrm{~s}^{-1}$ for 25007 and $331 \mathrm{~km} \mathrm{~s}^{-1}$ for 26087 , compared with 250 and $285 \mathrm{~km} \mathrm{~s}^{-1}$, respectively, before convolution. If we did not know the intrinsic profiles a priori and simply corrected for instrumental broadening by subtracting the widths in quadrature, we would have derived FWHM $=230 \mathrm{~km} \mathrm{~s}^{-1}$ for $\lambda 5007$ and $295 \mathrm{~km} \mathrm{~s}^{-1}$ for 26087 . That is, we would have underestimated the FWHM of $\lambda 5007$ and overestimated that of $\lambda 6087$. As in model 6, which produces profiles showing a correlation between FWHM and IP/ $n_{\text {cr }}$ (but the wings are no longer resolved!), FWHM correlations with IP would thus more likely result from low-resolution studies (e.g., De Robertis \&
Osterbrock 1986) than from high-resolution studies (e.g., Veilleux 1991a).

We have noted in $\S 5.3$ of Paper I that Mrk 79 and Mrk 704 are examples of objects that show a correlation of line width with ionization potential if the width is defined by a parameter that is more sensitive to extended profile wings than is the FWHM. We consider the $\left[\mathrm{O}_{\mathrm{I}}\right]$ and $\left[\mathrm{S}_{\mathrm{II}}\right]$ profiles of Mrk 704 to be "narrow" and that of $[\mathrm{Fe}$ VII] $\lambda 6087$ to be "broad" (Paper I), but in fact, all three profiles have similar FWHMs. We consider the [Fe VII] profile to be broad because of its extended blue wing, which significantly affects the interpercentile velocity at $20 \%$ parameter, IPV $(20 \%)$, used in Paper I. The above applies to models 4 and 5 . For the NLR corresponding to those models with wellresolved profile wings to show correlations of width with IP, the width must be defined by a parameter that is sensitive to extended profile wings rather than by the FWHM.

Model 7.-Model 7 (Fig. 7) illustrates the difficulty in constraining NLR models if we cannot infer a significant contribution to the emission lines from high-density gas (e.g., from [O $\mathrm{III}]$ and/or [Fe VII] density diagnostics). Without the constraint of both low and high density, we cannot rule out an NLR that encompasses only a narrow range in radial coordinate, if it nonetheless includes a substantial range in radial velocity. For example, such an NLR might include only a narrow range of ionization parameters, as required by the [Fe vII] $\lambda 6087$ and [Fe x] $\lambda 6374$ profiles, even with ionization parameter decreasing outward as $1 / r$.

In this model, $n \propto 1 / r(\Gamma \propto 1 / r)$ and $v \propto r$. The cloud electron density decreases outward from $10^{3}$ to $10^{2} \mathrm{~cm}^{-3}$, while $\Gamma$ decreases from 1.0 to 0.1 . The ionization parameter decreases by only one decade because this NLR encompasses only one decade in radial coordinate. Even this slight decrease in $\Gamma$ in the direction of increasing velocity (outward) has a discernible effect on the [Fe x] $\lambda 6374$ profile wings. However, the extended wings in all the other profiles are roughly consistent with those observed.

Model 8.-Model 8 (Fig. 8) shows the effects of increasing the maximum density by even one decade above the previous varying ionization parameter model. This corresponds to an extra decade in the range of radial coordinate and, more importantly, in the range of cloud velocity. Here $v_{\max } / v_{\min }=100$ (it was 10 for model 7), with the result that the profile wings are dominated by the low-velocity core. It is true in general that if this wide a range of velocity is included, the resulting profiles are very unforgiving of any deviation from uniformity in the emissivity law, and either wing-dominated or core-dominated profiles result (depending on whether the emissivity law accentuates the highvelocity or low-velocity contribution to the profiles).

It is important to note that we do not reject this model because the profiles are narrow but because almost all the flux is contained in the core. If this model NLR were observed at realistic $\mathbf{S} / \mathrm{N}$, one would infer $v_{\max }$ much less than $500 \mathrm{~km} \mathrm{~s}^{-1}$. If we renormalize the velocity field to, say, $v_{\max }=2500 \mathrm{~km} \mathrm{~s}^{-1}$, so that there is measurable flux at 500 $\mathrm{km} \mathrm{s}^{-1}$, then $v_{\min }$ is increased by a factor of 5 to $25 \mathrm{~km} \mathrm{~s}^{-1}$. The resulting profiles are 5 times broader but represent essentially the same model NLR with the property that, contrary to observations, the resulting profile wings are dominated by the core.

\section{RESULTS FOR MODELS WITH $v$ DECREASING OUTWARD}

Model 9.-Model 9 (Fig. 9) is a conventional gravitational inflow model $(\alpha=-0.5)$ with density and ionization 

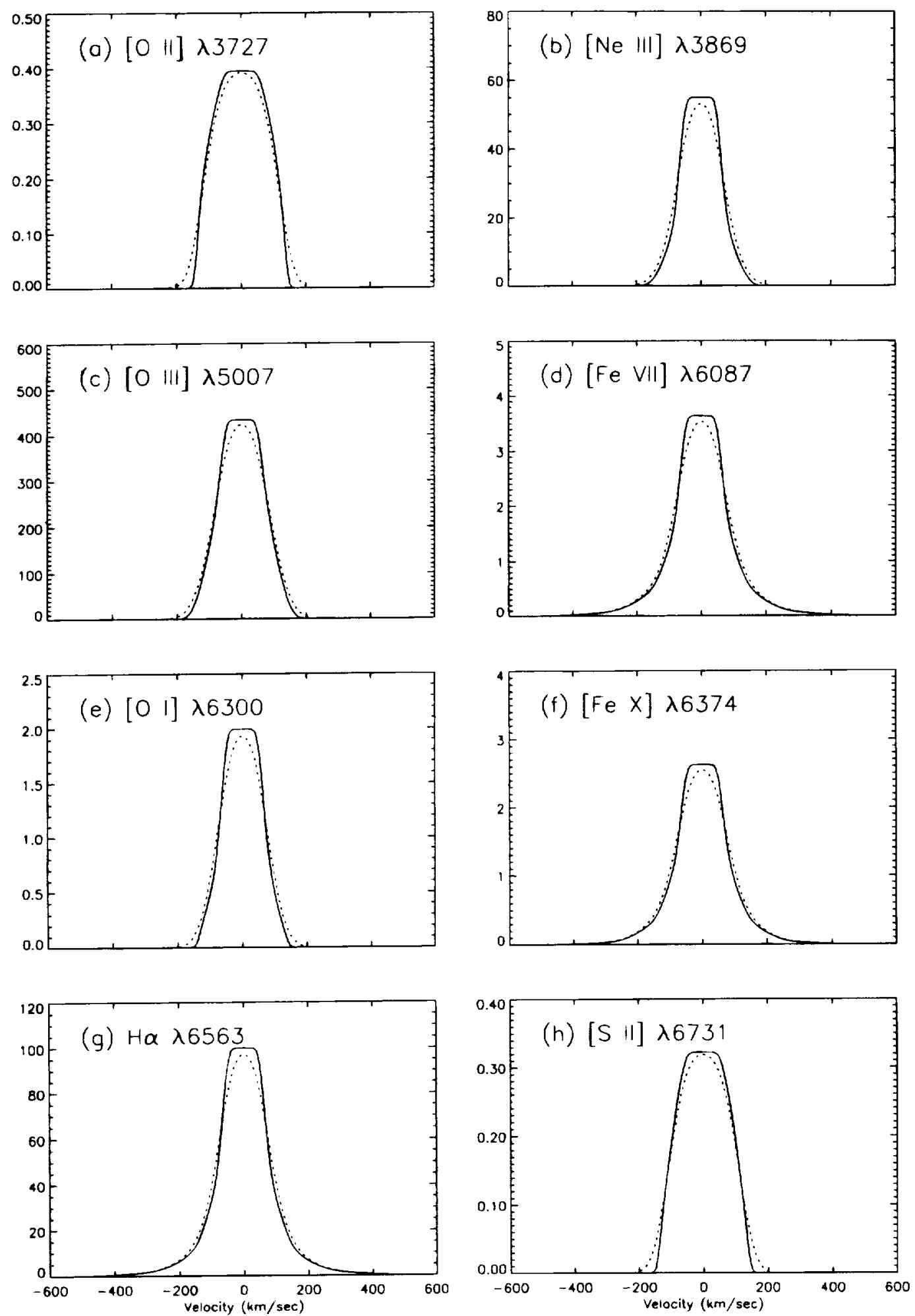

Fig. 4. - The line profiles of model 4, an outflow model. This model is identical to model 3 except that the cloud column density throughout the NLR is decreased by a factor of 4 . Specific model parameters are provided in Table 1 . We postulate that model 4 is representative of objects that show correlations of line width with ionization potential if the width is defined by a parameter that is sensitive to extended wings.

parameter decreasing outward as $1 / r$. The $[\mathrm{Fe}$ VII $] \lambda 6087$ profile is reasonable because the ionization parameter at the inner radius is 1.0 , which is rather high, and even though $\Gamma \propto 1 / r, \Gamma$ is sufficient to produce significant $[\mathrm{Fe} \mathrm{VII}] \lambda 6087$ emission throughout most of this NLR model. The wings of this profile are reasonable, in part, because $[\mathrm{Fe} \mathrm{VII}] \lambda 6087$ emission from the lowest velocity clouds with the lowest ionization parameter is weakened. The core of $\mathrm{H} \alpha$ is stronger relative to the wings than is the core of $[\mathrm{Fe}$ VII] $\lambda 6087$. Note that the synthetic $[\mathrm{Fe} \mathrm{x}] \lambda 6374$ line is not at all consistent with the profiles we have emphasized throughout this paper in either shape or strength. In addition, this model does not include $n \gtrsim 10^{6} \mathrm{~cm}^{-3}$ gas.

Model 10.-This model (Fig. 10) illustates the effects of 
No. 1, 1996
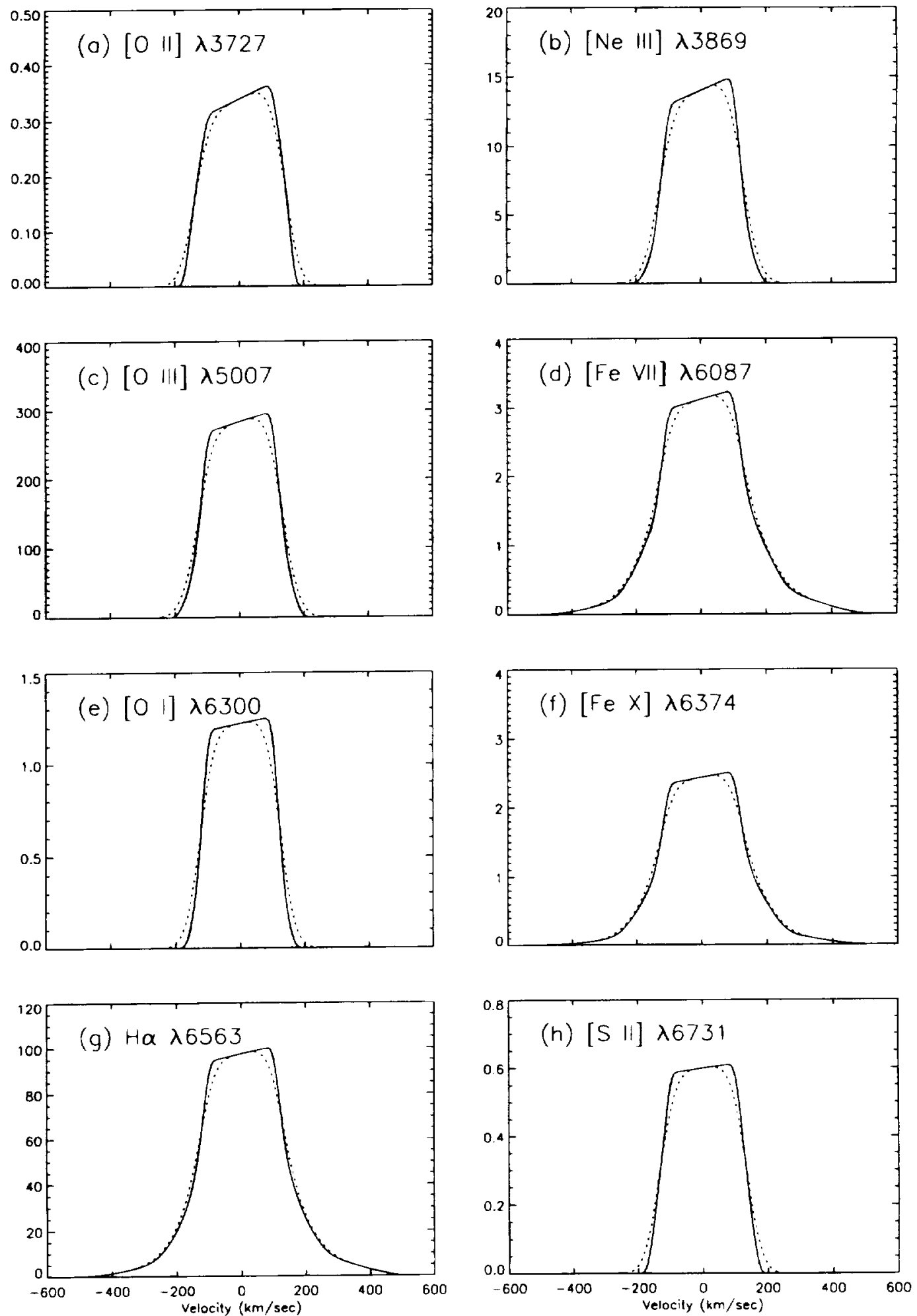

Fig. 5.- The line profiles of model 5, an outflow model. Specific model parameters are provided in Table 1 . This model is similar to model 4 but includes a smaller range of radial coordinate and velocity, resulting in a correlation of FWHM with ionization potential that is only barely discernible. In addition, due to the smaller range in radial coordinate, this model NLR does not include $n_{e} \geq 10^{6} \mathrm{~cm}^{-3}$ gas as required by the [O III] lines in many objects. We have also included obscuration by a dusty intercloud medium. The treatment of obscuration is identical to that of Vrtilek (1985)).

satisfying the constraints of both low and high density with a density profile decreasing as slowly as $1 / r$. The outer radius is comparable to that of an entire galaxy and is, therefore, completely unrealistic. The flux in the synthetic Fe lines is far lower than observations require, and the four decades of radial coordinate encompassed by this model NLR corresponds once again to $v_{\max } / v_{\min }=100$ so that, in the other lines, the profile core dominates the wings. Although this model produces profiles that are clearly inconsistent with those we have emphasized throughout 

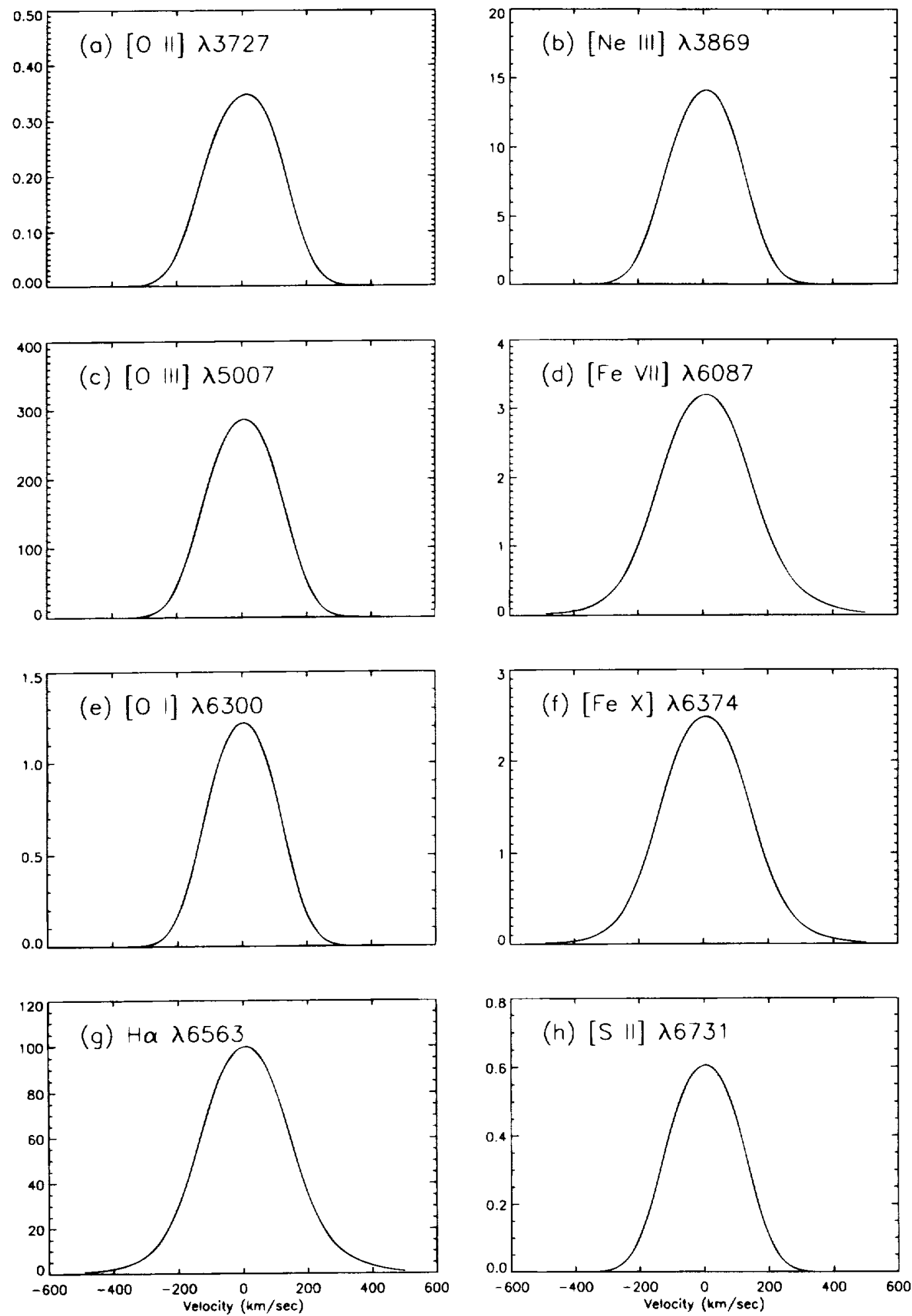

FiG. 6.-The line profiles of model 6, an outflow model. Model 6 is identical to model 5, but we have convolved the resulting profiles with a Gaussian with FWHM $=150 \mathrm{~km} \mathrm{~s}^{-1}(2.5 \AA$ at $5000 \AA)$ to illustrate the effects of lower spectral resolution. The extended wings in the line profiles of high ionization now have a discernible effect on the FWHM, as well as on a width parameter that is sensitive to extended profile wings.

this paper, the profiles may seem not too different from those of Mrk 841 ( $\$ 5.4$ of Paper I), provided we rescale the flux in the $\mathrm{Fe}$ lines by a large factor. However, a simple scaling of the Fe lines that has little effect on the other lines, is inconsistent with the large column density assumed in the model.

Model 11.-Model 11 (Fig. 11) is a gravitational inflow model $(\alpha=-0.5)$ with constant ionization parameter. We have already rejected this type of model in the discussion of the analytical models (MC) because the flux in the profile wings is dominated by that in the core.

Model 12.-Model 12 (Fig. 12) is the same as model 11 except that $N_{\text {col }}$ at the innermost radius has been decreased from $3 \times 10^{24} \mathrm{~cm}^{-2}$ to $10^{23} \mathrm{~cm}^{-2}$. Interestingly, this model 

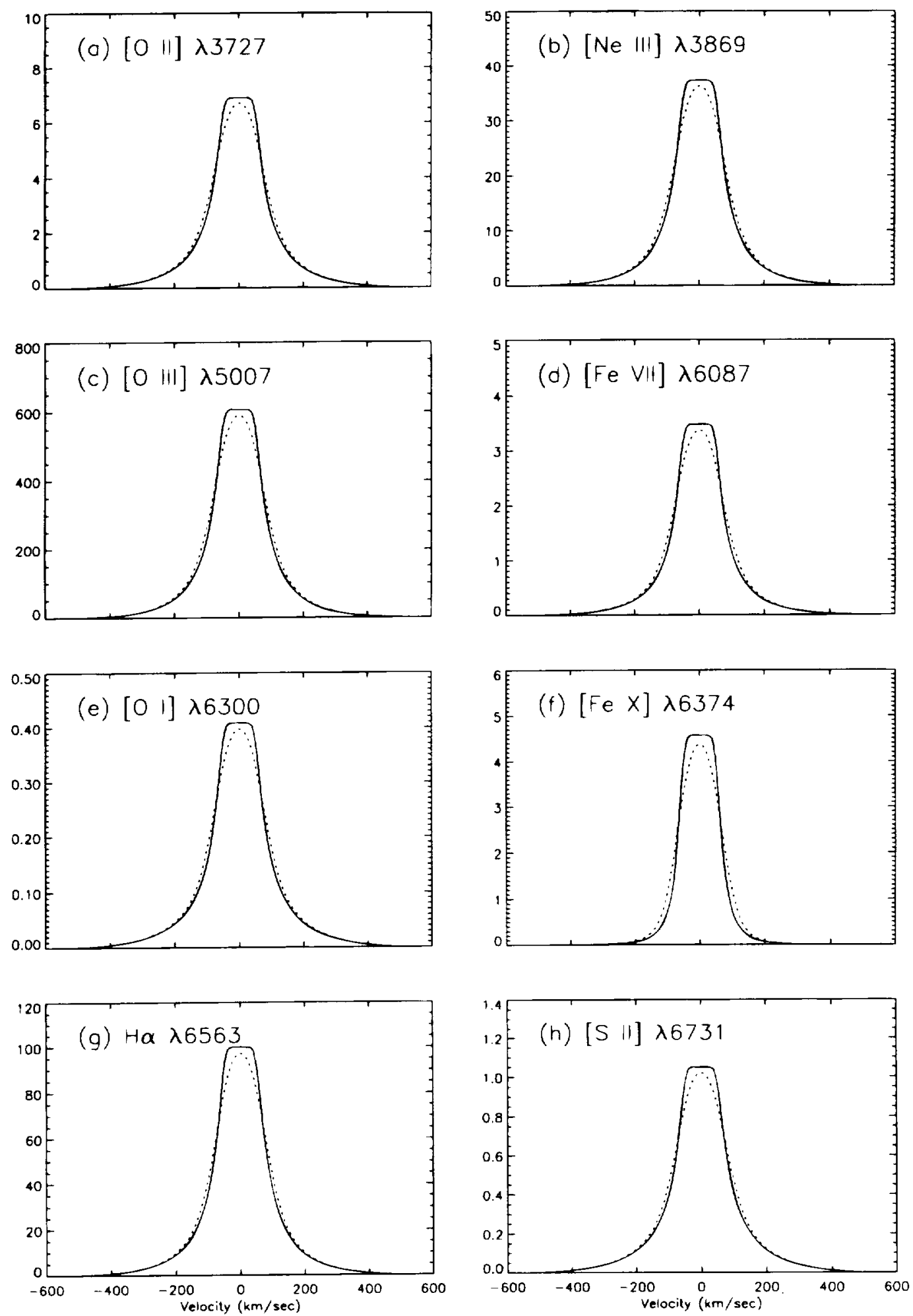

Fig. 7.-The line profiles of model 7, an outflow model. Specific model parameters are provided in Table 1. Model 7 illustrates the importance of constraining independently the range of radial coordinates and/or electron density included in the NLR. We reject this otherwise successful model only on the grounds that it violates constraints, inferred from the $[\mathrm{O} \mathrm{II}]$ lines and the $[\mathrm{S} \mathrm{II}]$ lines, on the range of electron density encompassed by the NLR.

yields extended profile wings in $[\mathrm{Fe} \mathrm{VII}] \lambda 6087,[\mathrm{Fe} \mathrm{x}]$ $\lambda 6374$, and $\mathrm{H} \alpha$ consistent with those observed. This is because $s / r_{\mathrm{fe}}$ decreases outward to one well before the outermost radius (minimum velocity) is reached, and this rapidly decreasing multiplicative factor diminishes the contribution to the profile from clouds of low velocity. This effect was not included in the analytical models, which assumed stratified clouds throughout the NLR. However, there is no emission into the other lines from the lower velocity-clouds with $s<r_{\mathrm{fe}}$, and profiles resembling boxcars result.

Model 13.-Model 13 (Fig. 13) is a compromise between gravitational inflow models that can account for both broad and narrow [O I] $\lambda 6300$ profiles $\left(n \propto r^{-6 / 5} ; N_{\text {col }} \approx\right.$ 

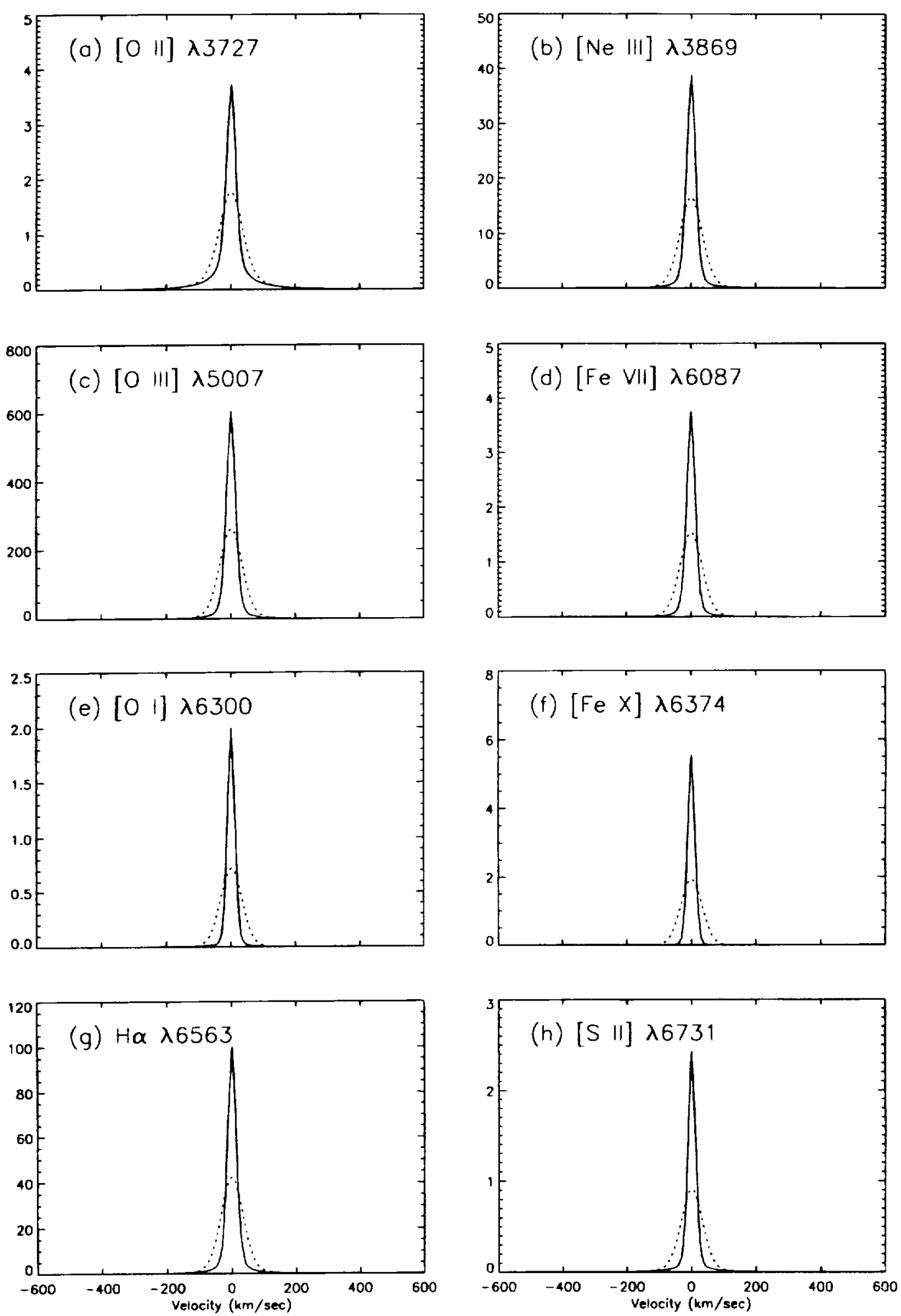

FIG. 8.-The line profiles of model 8 , an outflow model. Specific model parameters are provided in Table 1 . This model is similar to model 7 , but the range of density and radial coordinate and velocity is increased. This model illustrates that if the NLR includes a range of radial velocity as large as two decades, then it is difficult to produce profiles with comparable flux in the wings and core, as observations require. In model 8 , the profile wings are dominated by the low-velocity core.

$\left.N_{\min }[\mathrm{MC}]\right)$ and those favorable for strong $[\mathrm{Fe} \mathrm{VII}] \lambda 6087$ and $[\mathrm{Fe} \mathrm{x}] \lambda 6374$ emission throughout the NLR $\left(n \propto r^{-2}\right)$. In this model, the flux in the wings is weaker relative to the core than the profiles we have tried to replicate throughout this paper and in MC (with the exception of [O I] $\lambda 6300$ and $[\mathrm{Fe} \mathrm{x}] 26374)$, but perhaps not by so much that a similar model cannot be reconciled with observations by considering small variations in the parameters of this model, or perhaps by including secondary effects not included thus, far. Similarly, [Fe x] $\lambda 6734$ is weak compared to $[\mathrm{Fe}$ VII] $\lambda 6087$, but perhaps not hopelessly so. In addition, although we have argued that NLR gas encompasses a range of four decades in electron density (MC), the constraint is not so robust that we can properly rule out a 

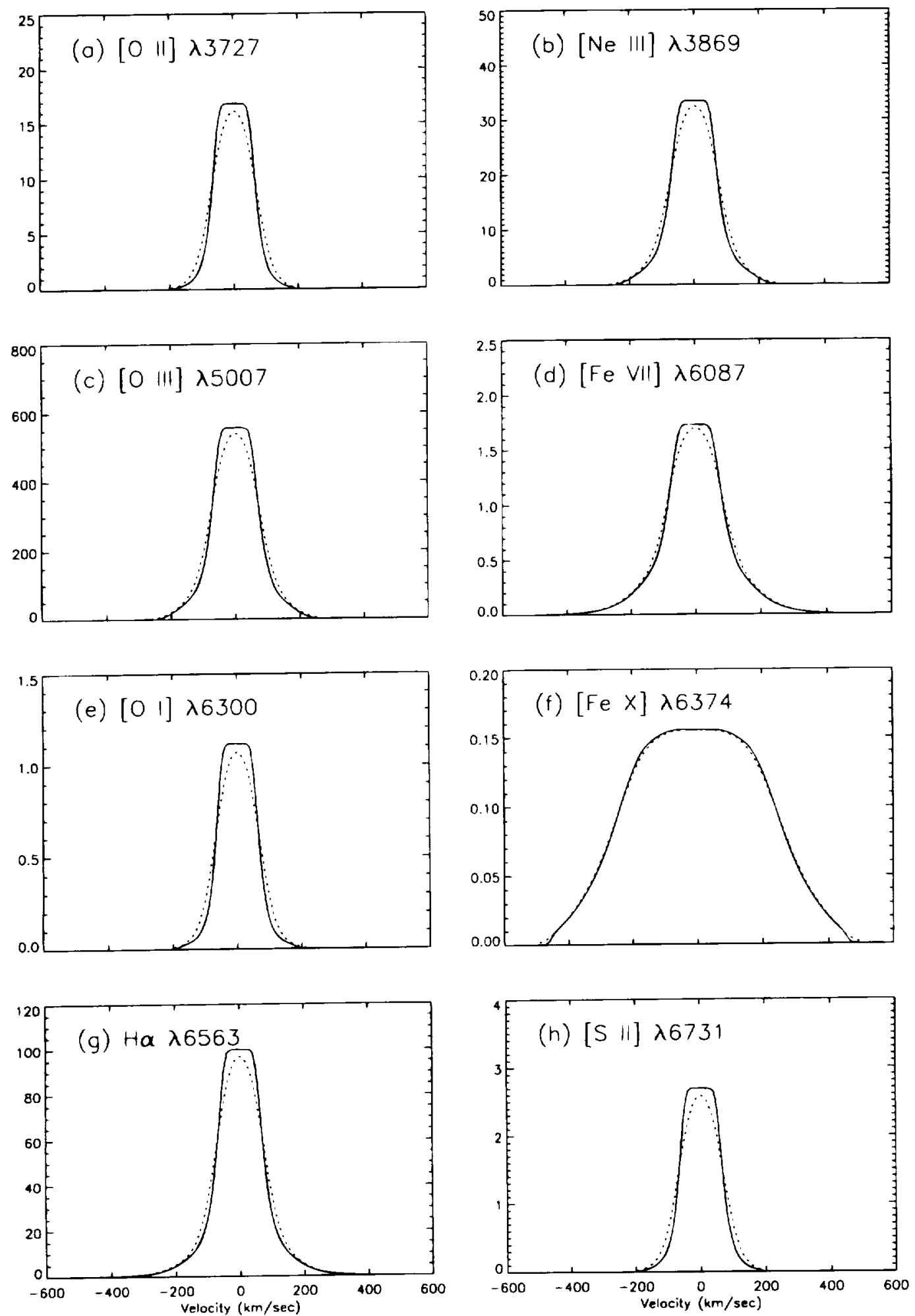

FIG. 9.-The line profiles of model 9. Specific model parameters are provided in Table 1. Model 9 is a classical gravitational inflow model with electron density and ionization parameter decreasing outward as $1 / r$, and with velocity decreasing outward as $r^{-0.5}$. The profiles of this model are not unreasonable except for that of $[\mathrm{Fe} \mathrm{X}] \lambda 6374$, which is especially sensitive to the decreasing ionization parameter. In addition, this model includes only low-density gas $\left(n_{\max }=10^{4} \mathrm{~cm}^{-3}\right)$, contrary to the $[\mathrm{O} \mathrm{III]}$ line strength ratios in many objects.

model that encompasses only three decades in electron density, as model 13 does. However, the parameters of model 13 are very contrived such that even though several attributes of the profiles are inconsistent with observations, no single attribute is more than marginally so. Only a narrow range of parameters can achieve this.

\section{SYNTHETIC LINE WIDTH CORRELATIONS}

In previous observational studies, a common approach to inferring the nature of the NLR velocity field has been to analyze line width correlations with IP and/or $n_{\text {cr }}$ (Osterbrock 1981; De Robertis \& Osterbrock 1984, 1986; 

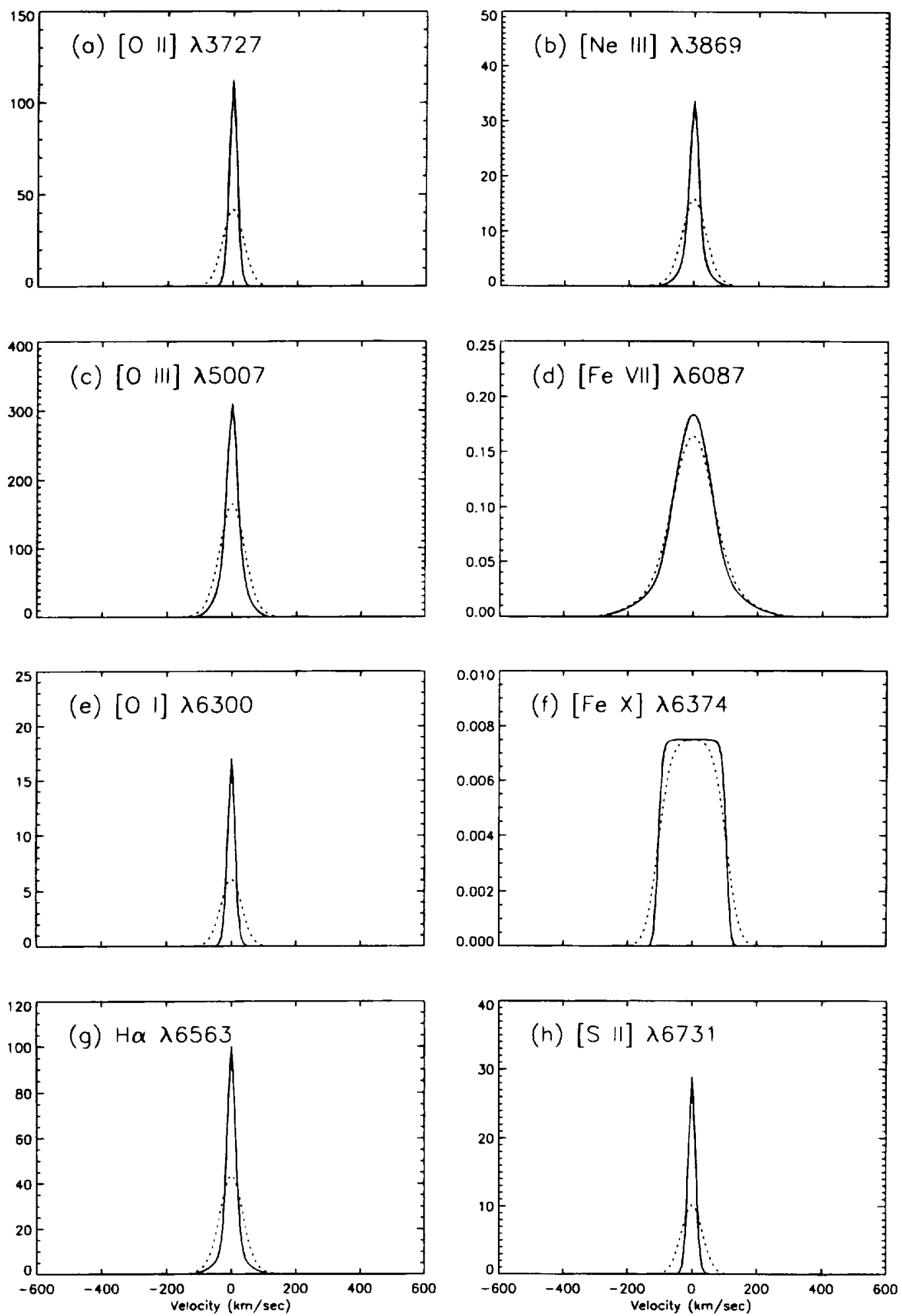

Fig. 10.-The line profiles of model 10, a gravitational inflow model. The parameters for this model are provided in Table 1 . Model 10 is similar to model 9 , but model 9 includes a very broad range of radial coordinates so that the constraints of both low- and high-density gas can be satisfied by a density law decreasing outward only as $1 / r$.

Whittle 1985b; Appenzeller \& Östreicher 1988; De Robertis \& Shaw 1990; Veilleux 1991b). The success of this approach depends on the qualitative assumption that lines of high $\mathrm{IP} / n_{\mathrm{cr}}$ are emitted more efficiently closer to the central source, relative to lines of low IP $/ n_{\mathrm{cr}}$. We have calculated explicitly synthetic profiles for a wide range of NLR models, and the predicted line width correlations resulting from our model profiles have some interesting implications, which we discuss in this section, for the observational studies enumerated above.

In Figure 14 we plot IPV (20\%) versus both $n_{\mathrm{cr}}$ and IP for models $3,4,9$, and $13(\S 3)$. Note the considerable scatter, 

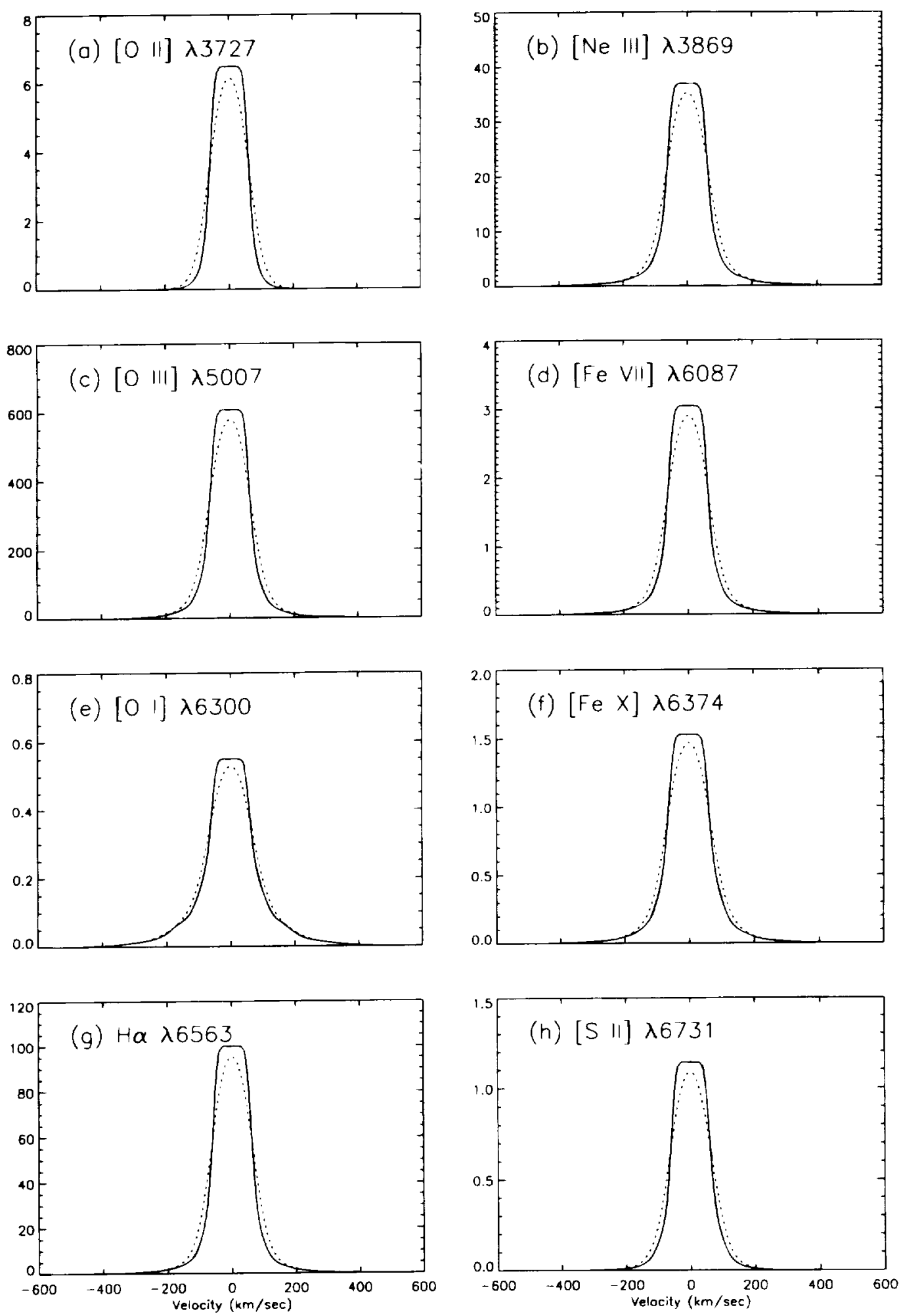

FIG. 11.-The line profiles of model 11, a gravitational inflow model with constant ionization parameter. Specific model parameters are provided in Table 1. We argue against this type of model because the flux in the profile wings is dominated by that in the core.

even with zero uncertainty in the measured widths. Although scatter is expected, even in synthetic correlation plots, because the assumption of a specific spread in cloud velocities corresponding directly to a specific IP and/or $n_{\mathrm{cr}}$ was never intended to apply more than qualitatively, the amount of scatter is greater than we would have anticipated. We noted in Paper I that our formal errors were well within the scatter in the widths calculated from our observed profiles.

As discussed in $\S 3$, we consider model 3 as a prototype of a model that shows correlations of line width with neither IP nor $n_{\mathrm{cr}}$. Model 4 , which differs from model 3 only in that the cloud column density is slightly smaller, is the prototype of a model that does show correlations of line width with 

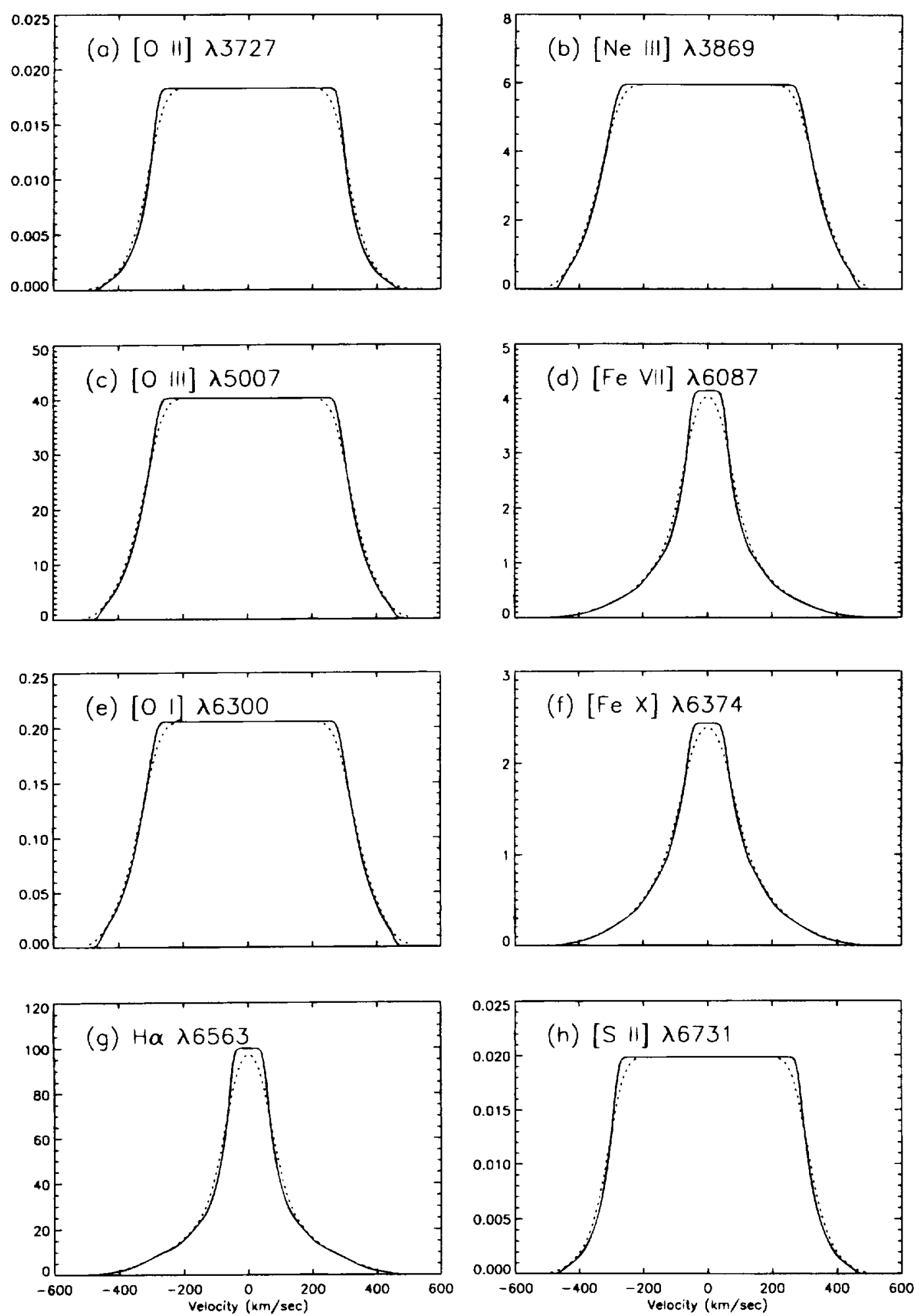

FIG. 12.-The line profiles of model 12. This model is identical to model 11 except that the cloud column density is decreased by a factor of 30 . Specific model parameters are provided in Table 1.

IP $/ n_{\mathrm{cr}}$. However, only if the widths of the lowest $n_{\mathrm{cr}}$ lines ([O II] $\lambda 3727$ and [S II] 26731 ) were decreased significantly would the line width plot for model 4 (or 3 ) roughly match correlations (or lack thereof) presented in observational studies. Here and in Paper I, we have already discussed why our favored models require emission from low-velocity and low-density gas outside the (parameter space of the) model NLR, so the dilemma posed by the large [O II] and [S II] line widths is entirely expected.

Model 9, a conventional gravitational inflow model with $n$ (and $\Gamma) \propto 1 / r$, yields the tightest correlation of line width with IP $/ n_{\text {cr }}$ of any model we have calculated. Indeed, the correlation of IPV $(20 \%)$ with IP is far tighter than any correlation we have seen in the literature. The greatest difficulty with model 9 is that the total flux in the [Fe] lines is far weaker than that observed, desite choosing a high ion- 

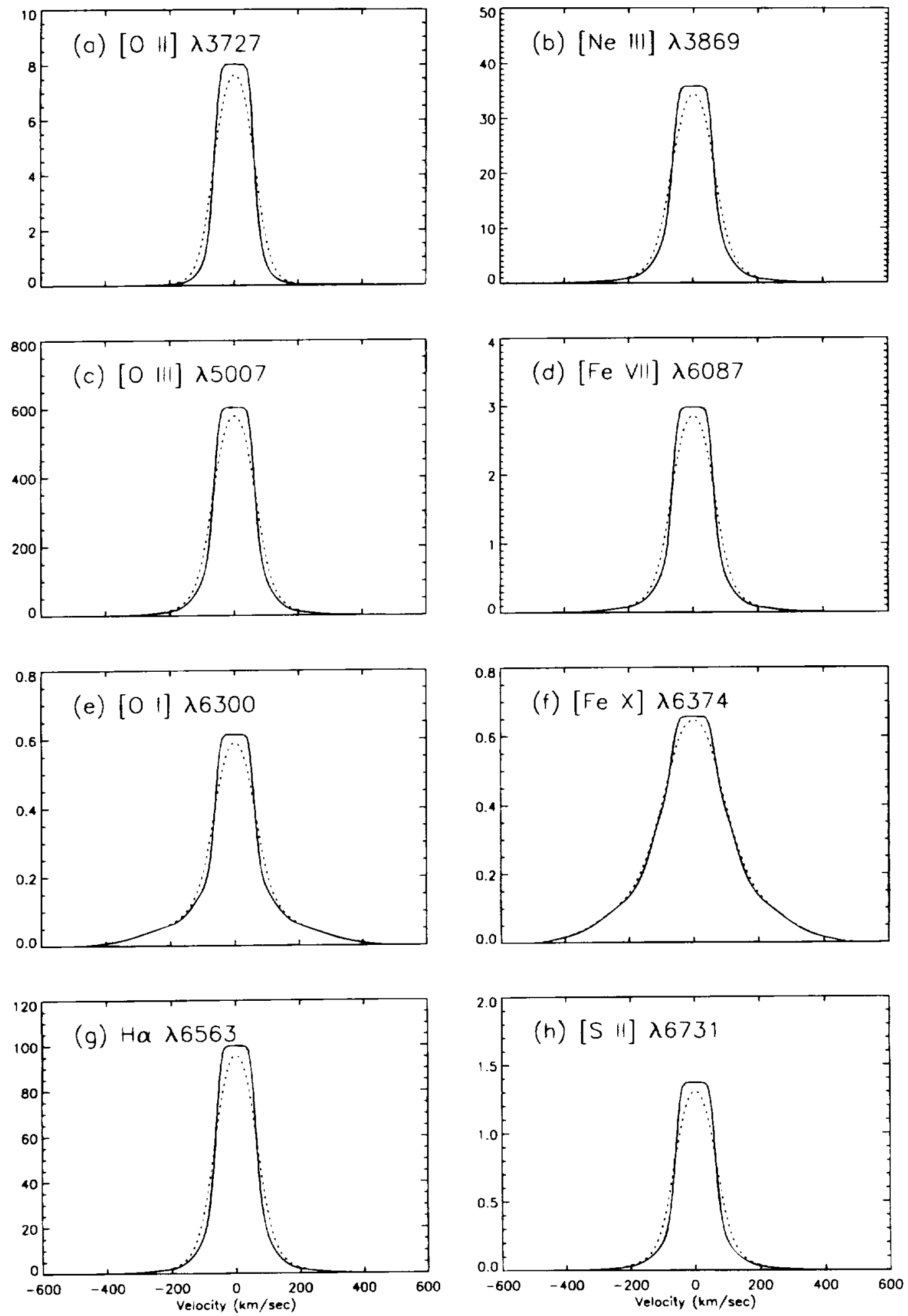

Fig. 13.-The line profiles of model 13, a gravitational inflow model. Specific model parameters are provided in Table 1 . This models is a compromise model in that several attributes of the synthetic profiles are inconsistent with observations, but no single attribute is more than marginally so. Only a narrow range of parameters can achieve this.

ization parameter specifically to minimize the difficulty. As was the case for [O II] and [S II] in models 3 and 4 , the [Fe] lines are broad (and weak) because the lowest velocity gas in the model does not emit these lines appreciably. Another disadvantage of model 9 is that a small change in the parameters could not account for an object that does not show line width correlations with $\mathrm{IP} / n_{\mathrm{cr}}$. Why we invoke low-velocity [O II] and [S II] emitting gas outside the model NLR to reconcile models 3 and 4 with observations when we could also invoke low-velocity $[\mathrm{Fe} \mathrm{VII}]$ and [Fe X]-emitting gas outside the model NLR to reconcile model 9 with observations is discussed further in $\S 6$.

In Figure 15 we plot the FWHM of emission lines versus both $n_{\mathrm{cr}}$ and IP for models 5 and 6 . These models are identi- 

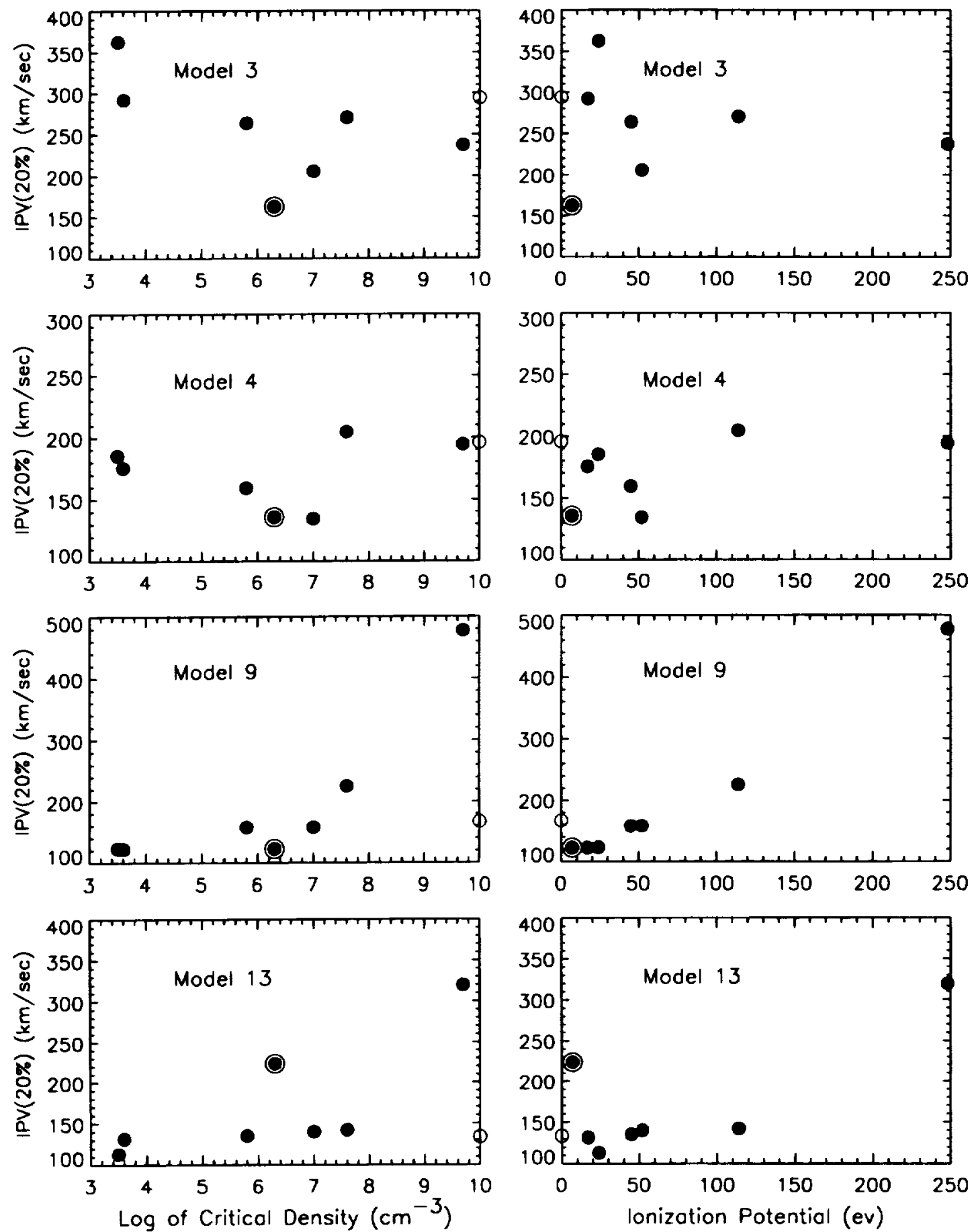

Fig. 14. - The width parameter of Whittle (1985a) (also used and discussed in Paper I), IPV (20\%), vs. both $n_{\mathrm{cr}}$ and IP for models $3,4,9$, and 13 . The model with the strongest line width correlations is model 9 , a conventional gravitational inflow model. Our favored models, 3 and 4, yield low $n_{\mathrm{cr}}$ line ([O $\left.\mathrm{n}\right] \lambda 3727$ and $[\mathrm{S} \mathrm{n}]$ ג6731) profiles that are broader (as well as weaker) than those observed.

cal except that model 6 has been convolved with a $\sigma=82$ $\mathrm{km} \mathrm{s}^{-1}$ Gaussian to simulate the effects of lower spectral resolution. Throughout this paper and Paper I, we have considered a profile broad if it shows extended wings. However, with high spectral resolution in which the extended wings are fully resolved (model 5), the profiles with and without extended wings have similar FWHM. Only after degrading the spectral resolution (model 6) do correlations of FWHM with IP $/ n_{\mathrm{cr}}$ result.

\section{DISCUSSION}

Some of the important results of these models support the more qualitative results of the analytical models discussed in MC. These results are summarized below. First we address some implicit biases in the conclusions inferred from these models, and we discuss related observational tests.

\subsection{Caveats and Related Observational Tests}

An NLR model that encompasses a substantial range of velocity over a narrow range of radial coordinate can yield profiles similar to those observed (e.g., models 1 and 7). It is difficult to constrain such a model. For example, as discussed here and in $\mathrm{MC}$, the forbidden $\mathrm{Fe}$ lines require a narrow range of ionization parameter, but this can be achieved even if $\Gamma \propto 1 / r$, provided $r_{\min } / r_{\max }$ is sufficiently 

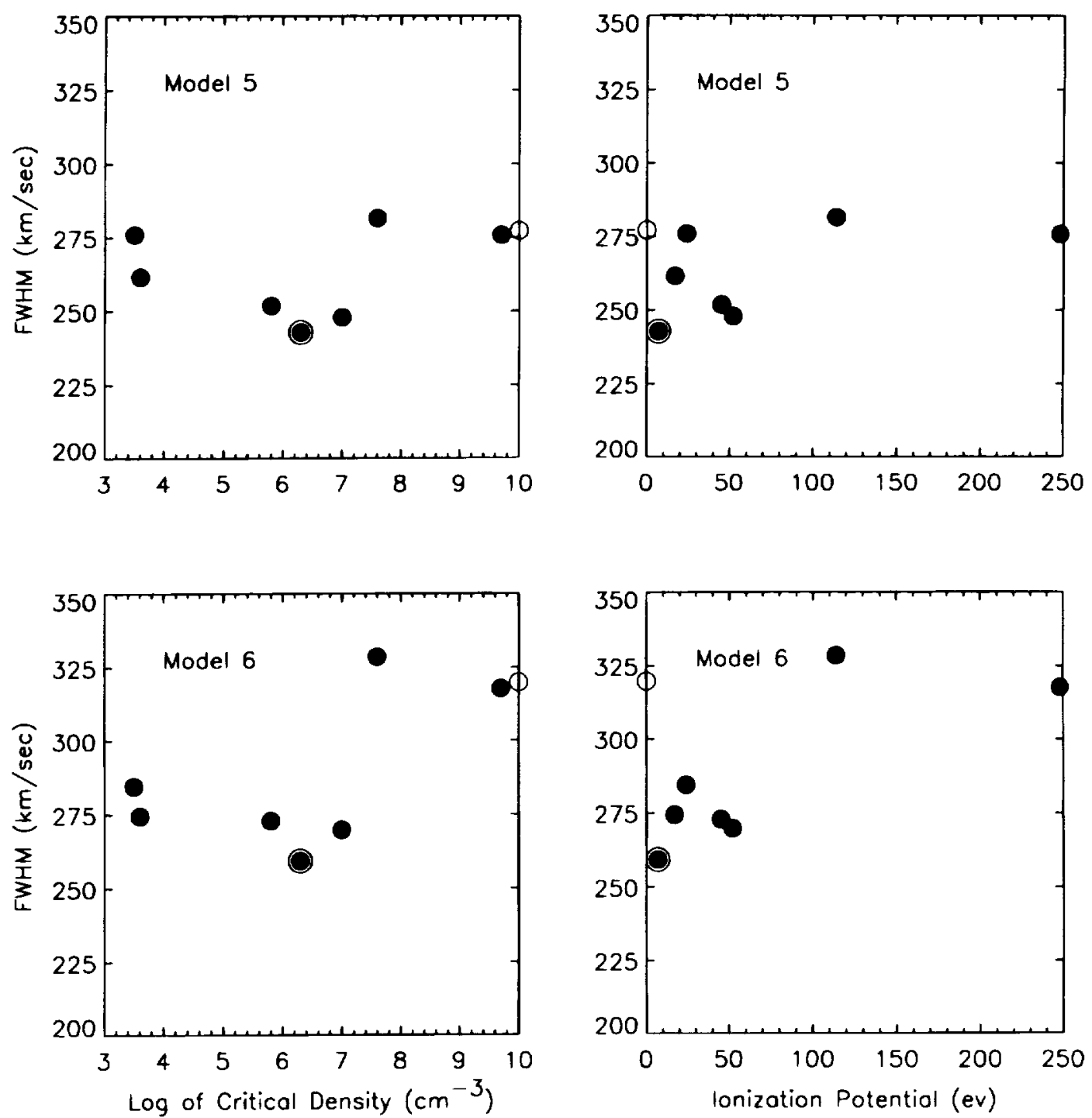

FIG. 15.-FWHM vs. both $n_{\mathrm{cr}}$ and IP for models 5 and 6 . These models are identical except that the profiles of model 6 have been convolved with a $\sigma=82$ $\mathrm{km} \mathrm{s}^{-1}(1.4 \AA$ at $5000 \AA)$ Gaussian to simulate the effects of lower spectral resolution. The most important effect is that conspicuous FWHM correlations result only after degrading the spectral resolution.

close to unity (see model 7). We can rule out this type of model only by invoking the constraints on the range of density (MC). Note, for example, that if we increase to four decades the range of density included in model 7 , then the resulting model will include four decades in ionization parameters, resulting in $\mathrm{Fe}$ line profiles very different from those of other lines (see, e.g., model 10). The constraint of high-density gas, inferred from the [O $\mathrm{OII}]$ lines, is not applicable to all objects, however. Nevertheless, we assume that the same physical processes are at work in objects for which we can and cannot infer the presence of high-density gas. Therefore, if the constraint of high density leads us to conclude that $v \propto r^{1 / 2}$ and $n_{e} \propto 1 / r^{2}$ in some objects, then we assume that these velocity and density laws are applicable in all objects. That is, we assume that objects for which we can and cannot infer the presence of high-density gas may differ in $n_{\max }$, but do not differ greatly in $v(r)$ and $n_{e}(r)$.

A related point is that in some objects, the increase in flux with decreasing projected velocity is more rapid than for the profiles we have emphasized throughout this paper (and MC). The most powerful profiles diagnostically are those with the most extended wings. When the distinction between wings and core is blurred, the observed profiles are consistent with a wide range of models. For example, the transition from wings to core is more abrupt in the [Fe vII] 26087 profile of Mrk 704 than of MCG 8-11-11 (Paper I) (thus, the distinction between wings and core is defined better in the former). The NLR models 5 and 6 are similar to model 3 except for a smaller $v_{\max } / v_{\min }$. If all NLRs are qualitatively similar, then we expect that the same acceleration mechanisms and basic assumptions apply to all objects if they apply to many objects, but differences in the line profiles from object to object can be explained, in part, by differences in $v_{\max } / v_{\min }$ (or equivalently, $r_{\max } / r_{\min }$ ) or perhaps by small differences in $N_{\text {col }}\left(r_{\min }\right)$.

Our favored NLR models (e.g., models 3 and 4) require the presence of low-density and low-velocity gas outside the model NLR to account for the observed profile cores of [O II] $\lambda 3727$ and [S II] $\lambda 6731$. The model profiles of these lines differ from those of the other lines because, in the superposition of boxcar profiles, the [O II] and [S II] profiles lack a contribution from the narrowest boxcar profiles due to the effects of collisional de-excitation at small $r$. However, the profile similarity observed in lines of both 
high and low $n_{\mathrm{cr}}$ extends into the profile cores in many objects (see, e.g., those of Veilleux 1991a). One might reasonably wonder why we invoke low-velocity $[\mathrm{O} \mathrm{II}]$ and $[\mathrm{S}$ II] emission outside our model to explain the $\lambda 3727$ and 26731 profiles when we could just as well invoke lowvelocity $[\mathrm{Fe} \mathrm{vil}]$ and $[\mathrm{Fe} \mathrm{x}]$ emission outside the model, perhaps relaxing the requirement of roughly constant ionization parameter throughout the NLR. One reason is that if gas outside our model produces low-velocity forbidden $\mathrm{Fe}$ line emission, this gas must still have an ionization parameter of about 0.3 (assuming photoionization, of course), which implies at least some form of continuity with the gas producing the high-velocity forbidden $\mathrm{Fe}$ line emission. [O II] $\lambda 3727$ and $\left[\mathrm{S}_{\mathrm{II}}\right] \lambda 6731$, on the other hand, are emitted over a wide range of ionization parameters. In fact, these lines are commonly emitted by gaseous nebulae heated by ordinary stars, while [Fe vII] $\lambda 6087$ and $[\mathrm{Fe} \mathrm{x}] \lambda 6374$ are usually associated with active galactic nuclei (AGNs).

The similarity of the profiles of lines with low and high $n_{\mathrm{cr}}$ in some objects seems fortuitous if gas outside the NLR model accounts for the core of the lowest $n_{\mathrm{cr}}$ lines but not for the core of the highest $n_{\mathrm{cr}}$ lines. However, the difficulty posed by the profile similarity is not avoided easily if constraints on the range of electron density encompassed by the NLR are taken seriously, and certainly not if the ionization parameter is roughly constant. The difficulty is also less severe if we note that we have already argued in MC that radial cloud flow does not by itself account for the profile cores and note also that our favoured model already requires some causal connection between the NLR and the host galaxy, other than gravitational influence by the latter, to explain various correlations of properties of NLR spectra with properties of the host galaxy (Busko \& Steiner 1988, 1989; Veilleux 1991c; Whittle 1992a, 1992b, 1992c; Nelson \& Whittle 1994). We argue for outflow in the NLR, which precludes gravity from either the host galaxy or from a central black hole as the dominant acceleration mechanism of NLR gas.

Considering that the [O II] $\lambda 3727$ profile core predicted by our favored model is inconsistent with observations, requiring emission from low-velocity and low-density gas outside the model NLR, the validity of the [O III] highdensity constraint is clearly worth careful attention. One important question is whether photoionization of highdensity gas is the most natural interpretation of relatively low values of $u \equiv(\lambda 4959+\lambda 5007) / \lambda 4363$ (such that $\lambda 5007$ and $\lambda 4959$ emission is suppressed relative to $\lambda 4363$ emission by collisional de-excitations). In Moore (1994), it was demonstrated that objects with relatively small $u$ tend also to have relatively small $\lambda 5007 / \mathrm{H} \beta$ line strength ratios, arguing (though not strongly) for photoionization of high-density gas rather than collisional ionization of lowdensity gas as the more likely interpretation of relatively small $u$.

A more direct test of the correct interpretation of small $u$ is to compare the [Fe vII $]$ line strength ratio, $(\lambda 5721+\lambda 6087) / \lambda 5159$, and the $[\mathrm{N} \mathrm{II}]$ line strength ratio, $(6548+\lambda 6584) / \lambda 5755$, with the [O III] line strength ratio. Each ratio is a useful diagnostic of $10^{5} \leqq n_{e} \leqslant 10^{7} \mathrm{~cm}^{-3}$ photoionized gas and is derived from lines fairly close in wavelength. It is unlikely that an alternative to photoionization could affect the [O III], [N II], and [Fe VII] density diagnostics in the same way. We are proposing a program of very high spatial resolution spectrophotometry of spa- tially resolvable NLRs on a previously unexplored angular scale using the Hubble Space Telescope (HST) in the optical as well as the Keck $10 \mathrm{~m}$ telescope in the mid-infrared. One of the goals of the $H S T$ observations is to isolate the contribution to spatially integrated emission-line spectra from the highest density clouds, obtaining $\mathrm{S} / \mathrm{N}$ sufficient to analyze the above lines which, though weak, are important diagnostics of electron density.

If the cloud electron density is constrained to a range such that collisional de-excitational is not important for [O II] $\lambda 3727$, then a constant ionization parameter model corresponds to a very narrow range of radial coordinate (factor of 10 or so). To sample a range of cloud velocity consistent with observed profiles would then require a steep velocity gradient; this would rule out gravitational inflow, which requires the velocity field to decrease as $r^{-1 / 2}$ or slower. However, if the NLR has a steep velocity gradient and small density range, we do not actually require a constant ionization parameter. Even with the ionization parameter decreasing outward, the $[\mathrm{Fe} \mathrm{VII}] \lambda 6087$ and $[\mathrm{Fe} \mathrm{x}] \lambda 6374$ profiles are similar to lines much less sensitive to ionization parameter because the range of ionization parameters is small. If relatively low values of $u \equiv(\lambda 4959+\lambda 5007) / \lambda 4363$ can be explained by an alternative to photoionization, then we cannot rule out such a model (see model 7). It is extremely important, therefore, to establish the correct interpretation of relatively small $u$.

If gas outside our model produces significant [S II] $\lambda \lambda 6716,6731$ emission, then the constraint of low density, inferred from the integrated flux in these lines, is not entirely valid. In this case, the constraint must be inferred from the high-velocity wings in these lines when they exist. Of course, extended wings in the $[\mathrm{S} \mathrm{II}]$ lines result in blended profiles. One of the goals of proposed HST observations is to obtain deblended [S II] $\lambda \lambda 6716,6731$ profiles in an object with extended $[\mathrm{S}$ II $]$ wings from spatially resolvable NLRs (e.g., NGC 4151, NGC 1068, and Mrk 3). If these observations can show that the NLR includes clouds with $n_{e} \approx 10^{2} \mathrm{~cm}^{-3}$ (from the [S II] lines) as well as clouds with $n_{e} \approx 10^{6} \mathrm{~cm}^{-3}$ (from the $[\mathrm{O} \mathrm{III}]$ and $[\mathrm{Fe} \mathrm{vII]} \mathrm{lines)} \mathrm{over} \mathrm{a} \mathrm{range} \mathrm{of} \mathrm{two}$ decades in radial coordinates, then the observations will lend further support to the assumption of constant ionization parameter. In this case, it is reasonable to assume that the low-velocity as well as high-velocity forbidden Fe line emission is from within the classical NLR even if this assumption implies that the low-velocity $[\mathrm{S} \mathrm{II}]$ and [O II] emission is from outside the classical NLR. The extra [S II] and $[\mathrm{O} \mathrm{II}]$ emission might come from $\mathrm{H}$ II regions or may even come from the outer parts of a dusty torus. The possibility of low-ionization forbidden-line emission from a torus warrants further study. For example, based on observed 21 $\mathrm{cm}$ absorption and on upper limits to molecular absorption, it has been calculated that a significant fraction of the gas in the dusty obscuring torus in Cygnus A could plausibly be in a hot $(5000-10,000 \mathrm{~K})$, mostly atomic phase (see Conway \& Blanco 1995, and references therein). We have assumed also that the high-velocity profile wings are emitted within the model NLR, whatever the implications for the low-velocity profile core. This is a justified bias in that many physical processes (some of which are enumerated in MC) certainly affect the profiles at the level of $v_{\min }$. More importantly, the observed blue asymmetry constrains the highest velocity clouds in a model NLR to move primarily in the radial direction regardless of the nature of the velocity field at low 
velocity. We have also shown (MC) that assuming spherical symmetry for a biconical NLR is valid for the profile wings, but not for the core.

\subsection{Summary of Conclusions}

We summarize now some of the important results of this work. In MC, we argued for constant ionization parameter based on the sensitivity of [Fe VII] $\lambda 6087$ and [Fe x] $\lambda 6374$ emission to the ionization parameter and the similarity of these profiles to those of lines much less sensitive to the ionization parameter. The modeling of this paper shows that if much NLR gas had an ionization parameter outside a range of about 0.1 to 1.0 , then the Fe line emission would differ in its spatial extent from the $\lambda 5007$ emission, for example, and, contrary to observations, Fe line profiles very different from those of $\lambda 5007$ would result.

Models 3 and 4 demonstrate that the highest velocity clouds must be optically thick to the photoionizing continuum in objects in which the $\left[\mathrm{O}_{\mathrm{I}}\right] \lambda 6300$ profile has similarly extended wings as the $[\mathrm{Fe}$ VII $] \lambda 6087$ profile. These two models also illustrate how objects that to and do not show line width correlations with ionization potential can both be explained by a small difference in cloud column density of the respective NLRs. In this scenario, $N_{\text {col }}$ decreases outward as $r^{-4 / 3}$ (constant mass spherical clouds with $n_{e} \propto$ $r^{-2}$ ) from typically $N_{\text {col }} \approx 10^{24} \mathrm{~cm}^{-2}$ to $N_{\text {col }} \approx 10^{22} \mathrm{~cm}^{-2}$.

Overall, the models presented here support the conclusion of $\mathrm{MC}$ that producing extended profile wings, which are often observed, requires that the velocity field increases outward if line emission is that of constant-mass spherical clouds that are conserved in number and have a constant ionization parameter. In our favored models, each radial coordinate, $r$, gives a relative contribution to the line profile proportional to $r^{1 / 3}$ which, though not steep, nevertheless precludes an arbitrarily wide range of cloud velocity from contributing to the model profiles if they are to be consistent with those observed. For a realistic range in radial coordinates of two decades or more, the velocity field must then increase outward as about $r^{0.5}$ rather than about $r^{1.0}$ so that comparable flux is emitted into the wings and the cores of the model profiles.

One can easily imagine physical processes that could invalidate our assumptions of cloud continuity and of constant cloud mass, especially since we argue for outflow so that the clouds and ICM need not be comoving. Regardless of these assumptions, however, what is required to produce profiles similar to those observed can be described simplistically as follows. The observed profiles cannot be replicated by the boxcar profile corresponding to a single velocity. A superposition of boxcar profiles, corresponding to a range of velocity, is required. The superposition must be such that neither the wings nor the core dominate the profile. That is, the flux in the wings is comparable to that in the core. A strictly uniform emissivity law can satisfy this condition even if the range of velocity is infinite $\left(v_{\min }=0\right)$. However, if a substantial range of velocity is included, the resulting profiles are very unforgiving of any deviation from uniformity in the emissivity law, and either wing-dominated or coredominated model profiles result (depending on whether the emissivity law accentuates the high-velocity or the lowvelocity contribution to the profiles). If the assumption of cloud continuity is altered drastically, then another assumption, such as that of constant cloud mass, must be modified in a contrived fashion to compensate such that the resulting emissivity law still favors neither the highest velocity clouds nor the lowest velocity clouds by a large factor.

Again, we thank Pam Capodicci for very helpful administrative assistance, and we also thank Ron Lyons for generous technical assistance in preparing the plots. It is also a pleasure to acknowledge useful discussions with V. T. Junkkarinen, G. W. Marcy, R. C. Puetter, and H. E. Smith. Again, we thank our anonymous referee for thoughtful review and for offering specific suggestions for improvements. This work has been supported by NASA grant NAG 5-1630.
Appenzeller, I., \& Östreicher, R. 1988, AJ, 95, 45

Busko, I. C., \& Steiner, J. E. 1988, MNRAS, 232, 525 1989. MNRAS, 238, 1479

Conway, J. E., \& Blanco, P. R. 1995, ApJ, 449, L131

De Robertis, M. M., \& Osterbrock, D. E. 1984, ApJ, 286, 171 1986, ApJ, 301, 727

De Robertis, M. M., \& Shaw, R. A. 1990, ApJ, 348, 421

Ferland, G. J. 1989, HAZY, A Brief Introduction to CLOUDY (OSU

Astron. Dept. Internal Rep. 89-001)

Moore, D. 1994, Ph.D. thesis, Univ. California, San Diego 1995 in preparation

Moore, D., \& Cohen, R. D. 1994, ApJ, 433, 602 (MC)

Moore, D., Cohen, R. D., \& Marcy, G. W. 1996, ApJ, 470, 000 (Paper I)

\section{REFERENCES}

Nelson, C. H., \& Whittle, M. 1994, BAAS, 25, 1337

Osterbrock, D. E. 1981, ApJ, 246, 696

Tsvetanov, Z. I., Kriss, G. A., Evans, I. N., \& Ford, H. C. 1994, BAAS, 26 966

Veilleux, S. 1991a, ApJS, 75, 357

1991b, ApJ, 368, 158

Vrtilek, J. M. 1985, ApJ, 294, 121

Whittle, M. 1982, Ph.D. thesis, Univ. Cambridge

1985a, MNRAS, 213, 1

- 1985b, MNRAS, 216,817

$1992 \mathrm{a}$, ApJS, 79,49

$1992 \mathrm{a}$, ApJS, 79, 49
$1992 \mathrm{~b}$, ApJ 387,109

1992c, ApJ, 387, 121

Note added in proof. - Since submission of these two papers, we have observed NGC 4151 with long-slit spectroscopy on the Keck $10 \mathrm{~m}$ telescope with 0.65 FWHM spatial resolution, comparable to the spatial resolution needed to test some of the predictions of both of these papers. For that object, the following predictions of these papers are confirmed:

1. The [Fe vII] $\lambda 6087$ and $[\mathrm{O} \mathrm{I}] \lambda 6300$ emission-line fluxes have comparable angular extents on the subarcsecond angular scale that partially resolves the source of most of the line emission.

2. Emission into the high-velocity wings of the $\left[\mathrm{S} \mathrm{II}_{\mathrm{I}}\right] \lambda 6716,6731$ profiles coincides spatially with that into the high-velocity wings of the $[\mathrm{Fe} \mathrm{VII}]$ and $[\mathrm{O} \mathrm{I}]$ profiles.

3. On the subarcsecond scale, the profiles of all three lines show a velocity shift that increases systematically with angular displacement from the nucleus, relative to the systemic velocity. 
